\title{
The genetic structure of the world's first farmers
}

\author{
Iosif Lazaridis ${ }^{1,2,} \uparrow$, Dani Nadel $^{3}$, Gary Rollefson ${ }^{4}$, Deborah C. Merrett ${ }^{5}$, Nadin Rohland ${ }^{1}$,
} Swapan Mallick $^{1,2,6}$, Daniel Fernandes ${ }^{7,8}$, Mario Novak ${ }^{7,9}$, Beatriz Gamarra ${ }^{7}$, Kendra Sirak ${ }^{7,10}$, Sarah Connell $^{7}$, Kristin Stewardson ${ }^{1,6}$, Eadaoin Harney ${ }^{1,6,11}$, Qiaomei $\mathrm{Fu}^{1,12,13}$, Gloria Gonzalez-Fortes $^{14}$, Songül Alpaslan Roodenberg ${ }^{15}$, György Lengyel ${ }^{16}$, Fanny Bocquentin ${ }^{17}$, Boris Gasparian $^{18}$, Janet M. Monge ${ }^{19}$, Michael Gregg ${ }^{19}$, Vered Eshed ${ }^{20}$, Ahuva-Sivan Mizrahi $^{20}$, Christopher Meiklejohn ${ }^{21}$, Fokke Gerritsen ${ }^{22}$, Luminita Bejenaru ${ }^{23}$, Matthias Blüher $^{24}$, Archie Campbell ${ }^{25}$, Gianpiero Cavalleri ${ }^{26}$, David Comas ${ }^{27}$, Philippe Froguel $^{28,29}$, Edmund Gilbert ${ }^{26}$, Shona M. Kerr ${ }^{25}$, Peter Kovacs ${ }^{30}$, Johannes Krause ${ }^{31,32,33}$, Darren McGettigan $^{34}$, Michael Merrigan ${ }^{35}$, D. Andrew Merriwether ${ }^{36}$, Seamus O'Reilly ${ }^{35}$, Martin B. Richards $^{37}$, Ornella Semino ${ }^{38}$, Michel Shamoon-Pour ${ }^{36}$, Gheorghe Stefanescu ${ }^{39}$, Michael Stumvoll $^{24}$, Anke Tönjes ${ }^{24}$, Antonio Torroni ${ }^{38}$, James F. Wilson ${ }^{40,41}$, Loic Yengo ${ }^{28,29}$, Nelli A. Hovhannisyan $^{42}$, Nick Patterson ${ }^{2}$, Ron Pinhasi ${ }^{7, *, \uparrow}$ and David Reich ${ }^{1,2,6, *, \dagger}$

*Co-senior authors;

$\dagger$ Correspondence and requests for materials should be addressed to:

I. L. (lazaridis@genetics.med.harvard.edu), R. P. (ron.pinhasi@ucd.ie),or D. R. (reich@genetics.med.harvard.edu)

${ }^{1}$ Department of Genetics, Harvard Medical School, Boston, Massachusetts 02115, USA

${ }^{2}$ Broad Institute of MIT and Harvard, Cambridge, Massachusetts 02142, USA

${ }^{3}$ The Zinman Institute of Archaeology, University of Haifa, Haifa 3498838, Israel

${ }^{4}$ Dept. of Anthropology, Whitman College, Walla Walla, WA 99362, USA

${ }^{5}$ Dept. of Archaeology, Simon Fraser University, Burnaby, British Columbia V5A 1S6, Canada

${ }^{6}$ Howard Hughes Medical Institute, Harvard Medical School, Boston, MA 02115, USA

${ }^{7}$ School of Archaeology and Earth Institute, Belfield, University College Dublin, Dublin 4, Ireland

${ }^{8}$ CIAS, Department of Life Sciences, University of Coimbra, Coimbra 3000-456, Portugal

${ }^{9}$ Institute for Anthropological Research, Zagreb 10000, Croatia

${ }^{10}$ Dept. of Anthropology, Emory University, Atlanta, Georgia 30322, USA

${ }^{11}$ Dept. of Organismic and Evolutionary Biology, Harvard University, Cambridge 02138, USA

${ }^{12}$ Dept. of Evolutionary Genetics, Max Planck Institute for Evolutionary Anthropology, Leipzig 04103, Germany

${ }^{13}$ Key Laboratory of Vertebrate Evolution and Human Origins of Chinese Academy of Sciences, IVPP, CAS, Beijing 100044, China

${ }^{14}$ Dept. of Biology and Evolution, University of Ferrara, Ferrara I-44121, Italy

${ }^{15}$ Independent Researcher, Santpoort-Noord, The Netherlands

${ }^{16}$ Department of Prehistory and Archaeology, University of Miskolc, 3515 MiskolcEgyetemváros, Hungary

${ }^{17}$ French National Centre for Scientific Research, UMR 7041, 92023 Nanterre Cedex, France

${ }^{18}$ Institute of Archaeology and Ethnology,National Academy of Sciences of the Republic of Armenia, 0025 Yerevan, Republic of Armenia

${ }^{19}$ University of Pennsylvania Museum of Archaeology and Anthropology, Philadelphia, PA 19104, USA

${ }^{20}$ Israel Antiquities Authority, Jerusalem 91710, Israel

${ }^{21}$ Dept. of Anthropology, University of Winnipeg, Winnipeg, Manitoba R3B 2E9, Canada 
${ }^{22}$ Netherlands Institute in Turkey, Istiklal Caddesi, Nur-i Ziya Sokak 5, Beyoğlu, Istanbul, Turkey

${ }^{23}$ Alexandru Ioan Cuza University of Iasi, Romania 700505, Romania

${ }^{24}$ Department of Internal Medicine and Dermatology, Clinic of Endocrinology and Nephrology, 04103 Leipzig, Germany

${ }^{25}$ Generation Scotland, Centre for Genomic and Experimental Medicine, MRC Institute of Genetics and Molecular Medicine, University of Edinburgh, Western General Hospital, Edinburgh EH4 2XU, Scotland

${ }^{26}$ RCSI Molecular \& Cellular Therapeutics, Royal College of Surgeons in Ireland, Dublin 2, Ireland

${ }^{27}$ Institut de Biologia Evolutiva (CSIC-UPF), Departament de Ciències Experimentals i de la Salut, Universitat Pompeu Fabra, 08003 Barcelona, Spain

${ }^{28}$ Univ. Lille, CNRS, Institut Pasteur de Lille, UMR 8199 - EGID, F-59000 Lille, France

${ }^{29}$ Department of Genomics of common disease, London Hammersmith Hospital, London W12 0HS, UK

${ }^{30}$ Leipzig University Medical Center, IFB AdiposityDiseases, 04103 Leipzig, Germany

${ }^{31}$ Max Planck Institute for the Science of Human History, 07745 Jena, Germany

${ }^{32}$ Institute for Archaeological Sciences, Archaeo- and Palaeogenetics, University of Tübingen, Tübingen, Germany

${ }^{33}$ Senckenberg Centre for Human Evolution and Palaeoenvironment, University of Tübingen, 72072 Tübingen, Germany

${ }^{34}$ Independent research, County Wicklow, Ireland

${ }^{35}$ Genealogical Society of Ireland, Dún Laoghaire, County Dublin, Ireland

${ }^{36}$ Department of Anthropology, Binghamton University, New York 13902, USA

${ }^{37}$ Department of Biological Sciences, School of Applied Sciences, University of Huddersfield, Queensgate, Huddersfield HD1 3DH, UK

${ }^{38}$ Dipartimento di Biologia e Biotecnologie "L. Spallanzani", Università di Pavia, 27100 Pavia, Italy

${ }^{39}$ Institutul de Cercetari Biologice, Iaşi 700505, Romania

${ }^{40}$ Usher Institute for Population Health Sciences and Informatics, University of Edinburgh, Edinburgh EH8 9AG, Scotland

${ }^{41}$ MRC Human Genetics Unit, MRC Institute of Genetics and Molecular Medicine, University of Edinburgh, Edinburgh EH4 2XU, Scotland

${ }^{42}$ Dept. of Ecology and Nature Protection, Yerevan State University, 0025 Yerevan, Republic of Armenia 
We report genome-wide ancient DNA from 44 ancient Near Easterners ranging in time between 12,000-1,400 BCE, from Natufian hunter-gatherers to Bronze Age farmers. We show that the earliest populations of the Near East derived around half their ancestry from a 'Basal Eurasian' lineage that had little if any Neanderthal admixture and that separated from other non-African lineages prior to their separation from each other. The first farmers of the southern Levant (Israel and Jordan) and Zagros Mountains (Iran) were strongly genetically differentiated, and each descended from local hunter-gatherers. By the time of the Bronze Age, these two populations and Anatolian-related farmers had mixed with each other and with the hunter-gatherers of Europe to drastically reduce genetic differentiation. The impact of the Near Eastern farmers extended beyond the Near East: farmers related to those of Anatolia spread westward into Europe; farmers related to those of the Levant spread southward into East Africa; farmers related to those from Iran spread northward into the Eurasian steppe; and people related to both the early farmers of Iran and to the pastoralists of the Eurasian steppe spread eastward into South Asia.

Between 10,000-9,000 BCE, humans began practicing agriculture in the Near East ${ }^{1}$. In the ensuing five millennia, plants and animals domesticated in the Near East spread throughout West Eurasia (a vast region that also includes Europe) and beyond. The relative homogeneity of present-day West Eurasians in a world context ${ }^{2}$ suggests the possibility of extensive migration and admixture that homogenized geographically and genetically disparate sources of ancestry. The spread of the world's first farmers from the Near East would have been a mechanism for such homogenization. To date, however, due to the poor preservation of DNA in warm climates, it has been impossible to study the population structure and history of the first farmers and to trace their contribution to later populations.

In order to overcome the obstacle of poor DNA preservation, we took advantage of two methodological developments. First, we sampled from the inner ear region of the petrous bone $^{3,4}$ that can yield up to $\sim 100$ times more endogenous DNA than other skeletal elements ${ }^{4}$. Second, we used in-solution hybridization ${ }^{5}$ to enrich extracted DNA for about 1.2 million single nucleotide polymorphism (SNP) targets ${ }^{6,7}$, making efficient sequencing practical by filtering out microbial and non-informative human DNA. We merged all sequences extracted from each individual, and randomly sampled a single sequence to represent each SNP, restricting to individuals with at least 9,000 SNPs covered at least once. We obtained genome-wide data passing quality control for 45 individuals on whom we had a median 
coverage of 172,819 SNPs (Methods). We assembled radiocarbon dates for 26 individuals (22 new generated for this study) (Supplementary Data Table 1).

The newly reported ancient individuals date to $\sim 12,000-1,400 \mathrm{BCE}$ and come from the southern Caucasus (Armenia), northwestern Anatolia (Turkey), Iran, and the southern Levant (Israel and Jordan) (Supplementary Data Table 1, Fig. 1a). (One individual had a radiocarbon date that was not in agreement with the date of its archaeological context and was also a genetic outlier.) The samples include Epipaleolithic Natufian hunter-gatherers from Raqefet Cave in the Levant (12,000-9,800 BCE); a likely Mesolithic individual from Hotu Cave in the Alborz mountains of Iran (probable date of 9,100-8,600 BCE); Pre-Pottery Neolithic farmers from 'Ain Ghazal and Motza in the southern Levant (8,300-6,700 BCE); and early farmers from Ganj Dareh in the Zagros mountains of western Iran (8,200-7,600 BCE). The samples also include later Neolithic, Chalcolithic ( 4,800-3,700 BCE), and Bronze Age ( 3,3501,400 BCE) individuals (Supplementary Information, section 1). We combined our data with previously published ancient data ${ }^{7,8,9,10,8,10-15}$ to form a dataset of 281 ancient individuals. We then further merged with 2,583 present-day people genotyped on the Affymetrix Human Origins array ${ }^{13,16}$ (238 new) (Supplementary Data Table 2; Supplementary Information, section 2). We grouped the ancient individuals based on archaeological culture and chronology (Fig. 1a; Supplementary Data Table 1). We refined the grouping based on patterns evident in Principal Components Analysis (PCA) ${ }^{17}$ (Fig. 1b; Extended Data Fig. 1), ADMIXTURE model-based clustering ${ }^{18}$ (Fig. 1c), and 'outgroup' $f_{3}$-analysis (Extended Data Fig. 2). We used $f_{4}$-statistics to identify outlier individuals and to cluster phylogenetically indistinguishable groups into ‘Analysis Labels' (Supplementary Information, section 3).

We analyzed these data to address six questions. (1) Previous work has shown that the first European farmers harboured ancestry from a Basal Eurasian lineage that diverged from the ancestors of north Eurasian hunter-gatherers and East Asians before they separated from each other ${ }^{13}$ What was the distribution of Basal Eurasian ancestry in the ancient Near East? (2) Were the first farmers of the Near East part of a single homogeneous population, or were they regionally differentiated? (3) Was there continuity between late pre-agricultural huntergatherers and early farming populations, or were the hunter-gatherers largely displaced by a single expansive population as in early Neolithic Europe? ${ }^{8}$ (4) What is the genetic contribution of these early Near Eastern farmers to later populations of the Near East? (5) What is the genetic contribution of the early Near Eastern farmers to later populations of 
mainland Europe, the Eurasian steppe, and to populations outside West Eurasia? (6) Do our data provide broader insights about population transformations in West Eurasia?

\section{Basal Eurasian ancestry was pervasive in the ancient Near East and associated with reduced Neanderthal ancestry}

The 'Basal Eurasians' are a lineage hypothesized ${ }^{13}$ to have split off prior to the differentiation of all other Eurasian lineages, including both eastern non-African populations like the Han Chinese, and even the early diverged lineage represented by the genome sequence of the $\sim 45,000$ year old Upper Paleolithic Siberian from Ust'-Ishim ${ }^{11}$. To test for Basal Eurasian ancestry, we computed the statistic $f_{4}$ (Test, Han; Ust'-Ishim, Chimp) (Supplementary Information, section 4), which measures the excess of allele sharing of Ust'-Ishim with a variety of Test populations compared to Han as a baseline. This statistic is significantly negative $(Z<-3.7)$ for all ancient Near Easterners as well as Neolithic and later Europeans, consistent with their having ancestry from a deeply divergent Eurasian lineage that separated from the ancestors of most Eurasians prior to the separation of Han and Ust'-Ishim. We used $q p A d m^{7}$ to estimate Basal Eurasian ancestry in each Test population. We obtain the highest estimates in the earliest populations from both Iran $(66 \pm 13 \%$ in the likely Mesolithic sample, $48 \pm 6 \%$ in Neolithic samples), and the Levant (44 $\pm 8 \%$ in Epipaleolithic Natufians) (Fig. 2), showing that Basal Eurasian ancestry was widespread across the ancient Near East.

West Eurasians harbour significantly less Neanderthal ancestry than East Asians ${ }^{19,20-23}$, which could be explained if West Eurasians (but not East Asians) have partial ancestry from a source diluting their Neandertal inheritance ${ }^{21}$. Supporting this theory, we observe a negative correlation between Basal Eurasian ancestry and the rate of shared alleles with Neanderthals ${ }^{19}$ (Supplementary Information, section 5; Fig. 2). By extrapolation, we infer that the Basal Eurasian population had lower Neanderthal ancestry than non-Basal Eurasian populations and possibly none (ninety-five percent confidence interval truncated at zero of 0-60\%; Fig. 2; Methods). The finding of little if any Neanderthal ancestry in Basal Eurasians could be explained if the Neanderthal admixture into modern humans 50,000-60,000 years ago ${ }^{11}$ largely occurred after the splitting of the Basal Eurasians from other non-Africans.

It is striking that the highest estimates of Basal Eurasian ancestry are from the Near East, given the hypothesis that it was there that most admixture between Neanderthals and modern humans occurred ${ }^{19,24}$. This could be explained if Basal Eurasians thoroughly admixed into the Near East before the time of the samples we analyzed but after the Neanderthal admixture. 
182 Alternatively, the ancestors of Basal Eurasians may have always lived in the Near East, but the lineage of which they were a part did not participate in the Neanderthal admixture.

A population without Neanderthal admixture, basal to other Eurasians, may have plausibly lived in Africa. Craniometric analyses have suggested that the Natufians may have migrated from north or sub-Saharan Africa ${ }^{25,26}$, a result that finds some support from Y chromosome analysis which shows that the Natufians and successor Levantine Neolithic populations carried haplogroup E, of likely ultimate African origin, which has not been detected in other ancient males from West Eurasia (Supplementary Information, section 6) ${ }^{7,8}$. However, no affinity of Natufians to sub-Saharan Africans is evident in our genome-wide analysis, as present-day sub-Saharan Africans do not share more alleles with Natufians than with other ancient Eurasians (Extended Data Table 1). (We could not test for a link to present-day North Africans, who owe most of their ancestry to back-migration from Eurasia ${ }^{27,28}$.) The idea of Natufians as a vector for the movement of Basal Eurasian ancestry into the Near East is also not supported by our data, as the Basal Eurasian ancestry in the Natufians ( $44 \pm 8 \%)$ is consistent with stemming from the same population as that in the Neolithic and Mesolithic populations of Iran, and is not greater than in those populations (Supplementary Information, section 4). Further insight into the origins and legacy of the Natufians could come from comparison to Natufians from additional sites, and to ancient DNA from north Africa.

\section{Extreme regional differentiation in the ancient Near East}

PCA on present-day West Eurasian populations (Methods) (Extended Data Fig. 1) on which we projected the ancient individuals (Fig. 1b) replicates previous findings of a Europe-Near East contrast along the horizontal Principal Component 1 (PC1) and parallel clines (PC2) in both Europe and the Near East (Extended Data Fig. 1) $7,8,13$. Ancient samples from the Levant project at one end of the Near Eastern cline, and ancient samples from Iran at the other. The two Caucasus Hunter Gatherers (CHG) ${ }^{9}$ are less extreme along PC1 than the Mesolithic and Neolithic individuals from Iran, while individuals from Chalcolithic Anatolia, Iran, and Armenia, and Bronze Age Armenia occupy intermediate positions. Qualitatively, the PCA has the appearance of a quadrangle whose four corners are some of the oldest samples:

210 bottom-left: Western Hunter Gatherers (WHG), top-left: Eastern Hunter Gatherers (EHG), 211 bottom-right: Neolithic Levant and Natufians, top-right: Neolithic Iran. This suggests the hypothesis that diverse ancient West Eurasians can be modelled as mixtures of as few as four 
213 streams of ancestry related to these populations, which we confirmed using qpWave

214 (Supplementary Information, section 7).

215 We computed squared allele frequency differentiation between all pairs of ancient West

216 Eurasians $^{29}$ (Methods; Fig. 3; Extended Data Fig. 3), and found that the populations at the

217 four corners of the quadrangle had differentiation of $\mathrm{F}_{\mathrm{ST}}=0.08-0.15$, comparable to the value

218 of 0.09-0.13 seen between present-day West Eurasians and East Asians (Han)

219 (Supplementary Data Table 3). In contrast, by the Bronze Age, genetic differentiation

220 between pairs of West Eurasian populations had reached its present-day low levels (Fig. 3):

221 today, $\mathrm{F}_{\mathrm{ST}}$ is $\leq 0.025$ for $95 \%$ of the pairs of West Eurasian populations and $\leq 0.046$ for all

222 pairs. These results point to a demographic process that established high differentiation

223 across West Eurasia and then reduced this differentiation over time.

Continuity between pre-farming hunter-gatherers and early farmers of the Near East

225 Our data document continuity across the hunter-gatherer / farming transition, separately in

226 the southern Levant and in the southern Caucasus-Iran highlands. The qualitative evidence

227 for this is that PCA, ADMIXTURE, and outgroup $f_{3}$ analysis cluster Levantine hunter-

228 gatherers (Natufians) with Levantine farmers, and Iranian and Caucasus Hunter Gatherers

229 with Iranian farmers (Fig. 1b; Extended Data Fig. 1; Extended Data Fig. 2). We confirm this

230 in the Levant by showing that its early farmers share significantly more alleles with Natufians

231 than with the early farmers of Iran: the statistic $f_{4}$ (Levant_N, Chimp; Natufian, Iran_N) is

232 significantly positive $(Z=13.6)$. The early farmers of the Caucasus-Iran highlands similarly

233 share significantly more alleles with the hunter-gatherers of this region than with the early

234 farmers from the Levant: the statistic $f_{4}$ (Iran_N, Chimp; Caucasus or Iran highland hunter-

235 gatherers, Levant_N) is significantly positive $(\mathrm{Z}>6)$.

How diverse first farmers of the Near East mixed to form the region's later populations

237 Almost all ancient and present-day West Eurasians have evidence of significant admixture

238 between two or more ancestral populations, as documented by statistics of the form $f_{3}($ Test;

239 Reference $_{1}$, Reference Re $_{2}$ which if negative, show that a Test population's allele frequencies

240 tend to be intermediate between two Reference populations ${ }^{16}$ (Extended Data Table 2). To

241 better understand the admixture history beyond these patterns, we used $q p A d m^{7}$, which can

242 evaluate whether a particular Test population is consistent with being derived from a set of

243 proposed source populations, and if so, infer mixture proportions (Methods). We used this 
approach to carry out a systematic survey of ancient West Eurasian populations to explore their possible sources of admixture (Fig. 4; Supplementary Information, section 7).

Among first farmers, those of the Levant trace $\sim 2 / 3$ of their ancestry to people related to Natufian hunter-gatherers and $\sim 1 / 3$ to people related to Anatolian farmers (Supplementary Information, section 7). Western Iranian first farmers cluster with the likely Mesolithic HotuIIIb individual and more remotely with hunter-gatherers from the southern Caucasus (Fig. 1b), and share alleles at an equal rate with Anatolian and Levantine early farmers (Supplementary Information, section 7), highlighting the long-term isolation of western Iran.

During subsequent millennia, the early farmer populations of the Near East expanded in all directions and mixed, as we can only model populations of the Chalcolithic and subsequent Bronze Age as having ancestry from two or more sources. The Chalcolithic people of western Iran can be modelled as a mixture of the Neolithic people of western Iran, the Levant, and Caucasus Hunter Gatherers (CHG), consistent with their position in the PCA (Fig. 1b). Admixture from populations related to the Chalcolithic people of western Iran had a wide impact, consistent with contributing $\sim 4 \%$ of the ancestry of Levantine Bronze Age populations in the south and $\sim 33 \%$ of the ancestry of the Chalcolithic northwest Anatolians in the west. Our analysis show that the ancient populations of the Chalcolithic Iran, Chalcolithic Armenia, Bronze Age Armenia and Chalcolithic Anatolia were all composed of the same ancestral components, albeit in slightly different proportions (Fig. 4b; Supplementary Information, section 7).

\section{The Near Eastern contribution to Europeans, East Africans and South Asians}

Admixture did not only occur within the Near East but extended towards Europe. To the north, a population related to people of the Iran Chalcolithic contributed $\sim 43 \%$ of the ancestry of early Bronze Age populations of the steppe. The spread of Near Eastern ancestry

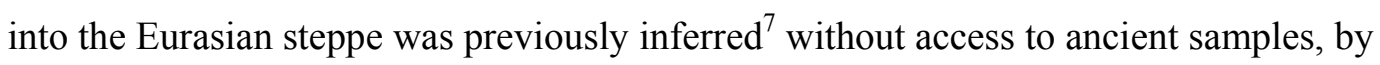
hypothesizing a population related to present-day Armenians as a source ${ }^{7,8}$. To the west, the early farmers of mainland Europe were descended from a population related to Neolithic northwestern Anatolians ${ }^{8}$. This is consistent with an Anatolian origin of farming in Europe, but does not reject other sources, since the spatial distribution of the Anatolian/European-like farmer populations is unknown. We can rule out the hypothesis that European farmers stem directly from a population related to the ancient farmers of the southern Levant ${ }^{30,31}$, however, 
275

276

277

278

279

280

281

282

283

since they share more allele with Anatolian Neolithic farmers than with Levantine farmers as attested by the positive statistic $f_{4}$ (Europe_EN, Chimp; Anatolia_N, Levant_N) $(\mathrm{Z}=15)$.

Migrations from the Near East also occurred towards the southwest into East African populations which experienced West Eurasian admixture $\sim 1,000 \mathrm{BCE}^{32,33}$. Previously, the West Eurasian population known to be the best proxy for this ancestry was present-day Sardinians $^{33}$, who resemble Neolithic Europeans genetically ${ }^{13,34}$. However, our analysis shows that East African ancestry is significantly better modelled by Levantine early farmers than by Anatolian or early European farmers, implying that the spread of this ancestry to East Africa was not from the same group that spread Near Eastern ancestry into Europe (Extended Data Fig. 4; Supplementary Information, section 8).

In South Asia, our dataset provides insight into the sources of Ancestral North Indians (ANI), a West Eurasian related population that no longer exists in unmixed form but contributes a variable amount of the ancestry of South Asians ${ }^{35,36}$ (Supplementary Information, section 9) (Extended Data Fig. 4). We show that it is impossible to model the ANI as being derived from any single ancient population in our dataset. However, it can be modelled as a mix of ancestry related to both early farmers of western Iran and to people of the Bronze Age Eurasian steppe; all sampled South Asian groups are inferred to have significant amounts of both ancestral types. The demographic impact of steppe related populations on South Asia was substantial, as the Mala, a south Indian population with minimal ANI along the 'Indian Cline' of such ancestry ${ }^{35,36}$ is inferred to have $\sim 18 \%$ steppe-related ancestry, while the Kalash of Pakistan are inferred to have $\sim 50 \%$, similar to present-day northern Europeans ${ }^{7}$.

\section{Broader insights into population transformations across West Eurasia and beyond}

We were concerned that our conclusions might be biased by the particular populations we happened to sample, and that we would have obtained qualitatively different conclusions without data from some key populations. We tested our conclusions by plotting the inferred position of admixed populations in PCA against a weighted combination of their inferred source populations and obtained qualitatively consistent results (Extended Data Fig. 5).

To further assess the robustness of our inferences, we developed a method to infer the existence and genetic affinities of ancient populations from unobserved 'ghost' populations (Supplementary Information, section 10; Extended Data Fig. 6). This method takes advantage of the insight that if an unsampled ghost population admixes with differentiated 'substratum' 
populations, it is possible to extrapolate its identity by intersecting clines of populations with variable proportions of 'ghost' and 'substratum' ancestry. Applying this while withholding major populations, we validated some of our key inferences, successfully inferring mixture proportions consistent with those obtained when the populations are included in the analysis. Application of this methods highlights the impact of Ancient North Eurasian (ANE) ancestry related to the $\sim 22,000 \mathrm{BCE}$ Mal'ta 1 and $\sim 15,000 \mathrm{BCE}$ Afontova Gora $2^{15}$ on populations living in Europe, the Americas, and Eastern Eurasia. Eastern Eurasians can be modelled as arrayed along a cline with different proportions of ANE ancestry (Supplementary Information, section 11; Extended Data Fig. 7), ranging from $\sim 40 \%$ ANE in Native Americans matching previous findings ${ }^{13,15}$, to no less than $\sim 5-10 \%$ ANE in diverse East Asian groups including Han Chinese (Extended Data Fig. 4; Extended Data Fig. 6f). We also document a cline of ANE ancestry across the east-west extent of Eurasia. Eastern Hunter Gatherers (EHG) derive $\sim 3 / 4$ of their ancestry from the ANE (Supplementary Information, section 11); Scandinavian hunter-gatherers ${ }^{7,8,13}$ (SHG) are a mix of EHG and WHG; and WHG are a mix of EHG and the Upper Paleolithic Bichon from Switzerland (Supplementary Information, section 7). Northwest Anatolians-with ancestry from a population related to European hunter-gatherers (Supplementary Information, section 7) - are better modelled if this ancestry is taken as more extreme than Bichon (Supplementary Information, section 10).

The population structure of the ancient Near East was not independent of that of Europe (Supplementary Information, section 4), as evidenced by the highly significant ( $Z=-8.9)$ statistic $f_{4}($ Iran_N, Natufian;WHG, EHG) which suggests gene flow in 'northeastern' (Neolithic Iran/EHG) and 'southwestern' (Levant/WHG) interaction spheres (Fig. 4d). This interdependence of the ancestry of Europe and the Near East may have been mediated by unsampled geographically intermediate populations ${ }^{37}$ that contribute ancestry to both regions.

\section{Conclusions}

By analysing genome-wide ancient DNA data from ancient individuals from the Levant, Anatolia, the southern Caucasus and Iran, we have provided a first glimpse of the demographic structure of the human populations that transitioned to farming. We reject the hypothesis that the spread of agriculture in the Near East was achieved by the dispersal of a single farming population displacing the hunter-gatherers they encountered. Instead, the spread of ideas and farming technology moved faster than the spread of people, as we can determine from the fact that the population structure of the Near East was maintained 
338 throughout the transition to agriculture. A priority for future ancient DNA studies should be

339 to obtain data from older periods, which would reveal the deeper origins of the population

340 structure in the Near East. It will also be important to obtain data from the ancient

341 civilizations of the Near East to bridge the gap between the region's prehistoric inhabitants

342 and those of the present. 


\section{Acknowledgements}

344 We thank the 238 human subjects who voluntarily donated the samples whose genome-wide

345 data we newly report in this study. We thank D. Labuda for sharing the collection of DNA

346 samples from Poland, and P. Zalloua for sharing the collection of DNA samples from

347 Lebanon. We thank O. Bar-Yosef, M. Bonogofsky, I. Hershkowitz, M. Lipson, I. Mathieson,

348 H. May, R. Meadow, I. Olalde, S. Paabo, P. Skoglund, and N. Nakatsuka for comments and

349 critiques, and M. Ferry and M. Michel for their work on the in-solution enrichment

350 experiments. S.C. was supported by the Irish Research Council for Humanities and Social

351 Sciences (IRCHSS) ERC Support Programme. Q.F. was funded by the Bureau of

352 International Cooperation of Chinese Academy of Sciences, the National Natural Science

353 Foundation of China (L1524016) and the Chinese Academy of Sciences Discipline

354 Development Strategy Project (2015-DX-C-03). The Scottish diversity data from Generation

355 Scotland received funding from the Chief Scientist Office of the Scottish Government Health

356 Directorates [CZD/16/6] and the Scottish Funding Council [HR03006], while the Scottish

357 Donor DNA Databank (GS:3D) was funded by a project grant from the Scottish Executive

358 Health Department, Chief Scientist Office [CZB/4/285]. M.S., A.Tön., M.B. and P.K. were

359 supported by grants from the Collaborative Research Center funded by the German Research

360 Foundation (CRC 1052; B01, B03, C01). M.S.-P. was partially funded by a Wenner-Gren

361 Foundation Dissertation Fieldwork Grant (\#9005), and by the National Science Foundation

362 DDRIG (BCS-1455744). P.K. was supported by the Federal Ministry of Education and

363 Research (BMBF), Germany (FKZ: 01EO1501). J.F.W acknowledges the MRC "QTL in

364 Health and Disease" programme grant. The Romanian contribution to this work was

365 supported by the EC Commission, Directorate General XII, within the framework of the

366 Cooperation in Science and Technology with Central and Eastern European Countries

367 (Supplementary Agreement ERBCIPDCT 940038 to the Contract ERBCHRXCT 920032,

368 coordinated by Prof. A. Piazza, Turin, Italy). M.R. received support from the Leverhulme

369 Trust's Doctoral Scholarship programme. O.S. and A.Tor. were supported by the University

370 of Pavia strategic theme "Towards a governance model for international migration: an

371 interdisciplinary and diachronic perspective" (MIGRAT-IN-G) and the Italian Ministry of

372 Education, University and Research: Progetti Ricerca Interesse Nazionale 2012. The Raqefet

373 Cave Natufian project was supported by funds from the National Geographic Society (Grant

374 \#8915-11), the Wenner-Gren Foundation (Grant \#7481) and the Irene Levi-Sala CARE

375 Foundation, while radiocarbon dating on the samples was funded by the Israel Science 
376 Foundation (Grant 475/10; E. Boaretto). R.P. was supported by ERC starting grant

377 ADNABIOARC (263441). D.R. was supported by NIH grant GM100233, by NSF

378 HOMINID BCS-1032255, and is a Howard Hughes Medical Institute investigator.

\section{Author Contributions}

380 R.P. and D.R. conceived the idea for the study. D.N., G.R., D.C.M., S.C., S.A., G.L., F.B., 381 B.Gas., J.M.M., M.G., V.E., A.M., C.M., F.G., N.A.H. and R.P. assembled archaeological 382 material. N.R., D.F., M.N., B.Gam., K.Si., S.C., K.St., E.H., Q.F., G.G.-F., R.P. and D.R. 383 performed or supervised ancient DNA wet laboratory work. L.B, M.B., A.C., G.C., D.C., 384 P.F., E.G., S.M.K., P.K., J.K., D.M., M.M., D.A.M., S.O., M.R., O.S., M.S.-P., G.S., M.S., 385 A.Tön., A.Tor., J.F.W., L.Y. and D.R. assembled present-day samples for genotyping. I.L, 386 N.P. and D.R. developed methods for data analysis. I.L., S.M., Q.F., N.P. and D.R. analyzed 387 data. I.L., R.P. and D.R. wrote the manuscript and supplements. All authors read the 388 manuscript and provided comments.

389 Author Information

390 The aligned sequences are available through the European Nucleotide Archive under 391 accession number xxx. Fully public subsets of the analysis datasets are at 392 (http://genetics.med.harvard.edu/reichlab/Reich_Lab/Datasets.html). The complete dataset 393 (including present-day humans for which the informed consent is not consistent with public 394 posting of data) is available to researchers who send a signed letter to D.R. indicating that 395 they will abide by specified usage conditions (Supplementary Information, section 2). 


\section{Online Methods}

\section{Ancient DNA data}

398 In a dedicated ancient DNA laboratory at University College Dublin, we prepared powder

399 from 132 ancient Near Eastern samples, either by dissecting the inner ear region of the

400 petrous bone using a sandblaster (Renfert), or by drilling using a Dremel tool and single-use

401 drill bits and selecting the best preserved bone fragments based on anatomical criteria. These

402 fragments were then powdered using a mixer mill (Retsch Mixer Mill 400) 4 .

403 We performed all subsequent processing steps in a dedicated ancient DNA laboratory at

404 Harvard Medical School, where we extracted DNA from the powder (usually $75 \mathrm{mg}$, range $14-81 \mathrm{mg}$ ) using an optimized ancient DNA extraction protocol ${ }^{38}$, but replaced the assembly of Qiagen MinElute columns and extension reservoirs from Zymo Research with a High Pure Extender Assembly from the High Pure Viral Nucleic Acid Large Volume Kit (Roche Applied Science). We built a total of 170 barcoded double-stranded Illumina sequencing libraries for these samples ${ }^{39}$, of which we treated 167 with Uracil-DNA glycosylase (UDG) to remove the characteristic C-to-T errors of ancient DNA ${ }^{40}$. The UDG treatment strategy is (by-design) inefficient at removing terminal uracils, allowing the mismatch rate to the human genome at the terminal nucleotide to be used for authentication ${ }^{39}$. We updated this library preparation protocol in two ways compared to the original publication: first, we used $16 \mathrm{U}$ Bst2.0 Polymerase, Large Fragment (NEB) and 1x Isothermal Amplification buffer (NEB) in a final volume of $25 \mu \mathrm{L}$ fill-in reaction, and second, we used the entire inactivated $25 \mu \mathrm{L}$ fill-in reaction in a total volume of $100 \mu \mathrm{L}$ PCR mix with $1 \mu \mathrm{M}$ of each primer ${ }^{41}$. We included extraction negative controls (where no sample powder was used) and library negative controls (where extract was supplemented by water) in every batch of samples processed and carried them through the entire wet lab processing to test for reagent contamination.

We screened the libraries by hybridizing them in solution to a set of oligonucleotide probes tiling the mitochondrial genome ${ }^{42}$, using the protocol described previously ${ }^{7}$. We sequenced

422 the enriched libraries using an Illumina NextSeq 500 instrument using $2 \times 76 \mathrm{bp}$ reads,

423 trimmed identifying sequences (seven base pair molecular barcodes at either end) and any

424 trailing adapters, merged read pairs that overlapped by at least 15 base pairs, and mapped the

425 merged sequences to the RSRS mitochondrial DNA reference genome ${ }^{43}$, using the Burrows

426 Wheeler Aligner ${ }^{44}(b w a)$ and the command samse (v0.6.1). 
427 We enriched promising libraries for a targeted set of $\sim 1.2$ million SNPs $^{8}$ as in ref. 5, and

428 adjusted the blocking oligonucleotide and primers to be appropriate for our libraries. The

429 specific probe sequences are given in Supplementary Data 2 of ref. 7

430 (http://www.nature.com/nature/journal/v522/n7555/abs/nature14317.html\#supplementary-

431 information) and Supplementary Data 1 of ref. 6.

432 (http://www.nature.com/nature/journal/v524/n7564/full/nature14558.html\#supplementary-

433 information). We sequenced the libraries on an Illumina NextSeq 500 using $2 \times 76 \mathrm{bp}$ reads.

434 We trimmed identifying sequences (molecular barcodes) and any trailing adapters, merged

435 pairs that overlapped by at least 15 base pairs (allowing up to one mismatch), and mapped the

436 merged sequences to hgl9 using the single-ended aligner samse in bwa (v0.6.1). We

437 removed duplicated sequences by identifying sets of sequences with the same orientation and

438 start and end positions after alignment to $h g 19$; we picked the highest quality sequence to

439 represent each set. For each sample, we represented each SNP position by a randomly chosen

440 sequence, restricting to sequences with a minimum mapping quality (MAPQ $\geq 10$ ), sites with a

441 minimum sequencing quality $(\geq 20)$, and removing 2 bases at the ends of reads. We sequenced

442 the enriched products up to the point that we estimated that generating a hundred new

443 sequences was expected to add data on less than about one new $\mathrm{SNP}^{8}$.

\section{Testing for contamination and quality control}

445 For each ancient DNA library, we evaluated authenticity in several ways. First, we estimated

446 the rate of matching to the consensus sequence for mitochondrial genomes sequenced to a

447 coverage of at least 10-fold from the initial screening data. Of the 76 libraries that contributed

448 to our dataset (coming from 45 samples), 70 had an estimated rate of sequencing matching to

449 the consensus of $>95 \%$ according to contamMix ${ }^{5}$ (the remaining libraries had estimated

450 match rates of $75-92 \%$, but gave no sign of being outliers in principal component analysis or

$451 \mathrm{X}$ chromosome contamination analysis so we retained them for analysis) (Supplementary

452 Data Table 1). We quantified the rate of C-to-T substitution in the final nucleotide of the

453 sequences analyzed, relative to the human reference genome sequence, and found that all the

454 libraries analyzed had rates of at least $3 \%{ }^{39}$, consistent with genuine ancient DNA. For the

455 nuclear data from males, we used the ANGSD software ${ }^{45}$ to estimate a conservative $\mathrm{X}$

456 chromosome estimate of contamination. We determined that all libraries passing our quality

457 control and for which we had sufficient X chromosome data to make an assessment had

458 contamination rates of $0-1.5 \%$. Finally, we merged data for samples for which we had

459 multiple libraries to produce an analysis dataset. 


\section{Affymetrix Human Origins genotyping data}

461 We genotyped 238 present-day individuals from 17 diverse West Eurasian populations on the

462 Affymetrix Human Origins array ${ }^{16}$, and applied quality control analyses as previously

463 described $^{13}$ (Supplementary Data Table 2). We merged the newly generated data with data

464 from 2,345 individuals previously genotyped on the same array ${ }^{13}$. All individuals that were

465 genotyped provided informed consent consistent with studies of population history, following

466 protocols approved by the ethical review committees of the institutions of the researchers

467 who collected the samples. De-identified aliquots of DNA from all individuals were sent to

468 the core facility of the Center for Applied Genomics at the Children's Hospital of

469 Philadelphia for genotyping and data processing. For 127 of the individuals with newly

470 reported data, the informed consent was consistent with public distribution of data, and the

471 data can be downloaded at http://genetics.med.harvard.edu/reich/Reich_Lab/Datasets.html.

472 To access data for the remaining 111 samples, researchers should a signed letter to D.R.

473 containing the following text: "(a) I will not distribute the samples marked "signed letter"

474 outside my collaboration; (b) I will not post data from the samples marked "signed letter"

475 publicly; (c) I will make no attempt to connect the genetic data for the samples marked

476 "signed letter" to personal identifiers; (d) I will not use the data for samples marked "signed

477 letter" for commercial purposes." Supplementary Data Table 2 specifies which samples are

478 consistent with which type of data distribution.

\section{Datasets}

480 We carried out population genetic analysis on two datasets: (i) $H O$ includes 2,583 present-

481 day humans genotyped on the Human Origins array ${ }^{13,16}$ including 238 newly reported

482 (Supplementary Data Table 2; Supplementary Information, section 2), and 281 ancient

483 individuals on a total of 592,146 autosomal SNPs. (ii) HOIll includes the 281 ancient

484 individuals on a total of 1,055,186 autosomal SNPs, including those present in both the

485 Human Origins and Illumina genotyping platforms, but excluding SNPs on the sex

486 chromosomes or additional SNPs of the 1240k capture array that were included because of

487 their potential functional importance ${ }^{8}$. We used $H O$ for analyses that involve both ancient and

488 present-day individuals, and HOIll for analysis on ancient individuals alone. We also use 235

489 individuals from Pagani et al. ${ }^{32}$ on 418,700 autosomal SNPs to study admixture in East

490 Africans (Supplementary Information, section 8). Ancient individuals are represented in

491 'pseudo-haploid' form by randomly choosing one allele for each position of the array. 


\section{Principal Components Analysis}

493 We carried out principal components analysis in the smartpca program of EIGENSOFT ${ }^{17}$,

494 using default parameters and the lsqproject: YES $^{13}$ and numoutlieriter: 0 options. We carried 495 out PCA of the $H O$ dataset on 991 present-day West Eurasians (Extended Data Fig. 1), and 496 projected the 278 ancient individuals (Fig. 1b).

\section{ADMIXTURE Analysis}

498 We carried out ADMIXTURE analysis ${ }^{18}$ of the $H O$ dataset after pruning for linkage 499 disequilibrium in PLINK ${ }^{46,47}$ with parameters --indep-pairwise 200250.4 which retained 500296,309 SNPs. We performed analysis in 20 replicates with different random seeds, and 501 retained the highest likelihood replicate for each value of $\mathrm{K}$. We show the $\mathrm{K}=11$ results for 502 the 281 ancient samples in Fig. 1c (this is the lowest $\mathrm{K}$ for which components maximized in 503 European hunter-gatherers, ancient Levant, and ancient Iran appear).

505 We carried out analysis of $f_{3}$-statistics, $f_{4}$-ratio, and $f_{4}$-statistics statistics using the 506 ADMIXTOOLS ${ }^{16}$ programs qp3Pop, qpF4ratio with default parameters, and qpDstat with 507 f4mode: YES, and computed standard errors with a block jackknife ${ }^{48}$. For computing $f_{3^{-}}$ 508 statistics with an ancient population as a target, we set the inbreed:YES parameter. We 509 computed $f$-statistics on the HOIll dataset when no present-day humans were involved and on 510 the $H O$ dataset when they were. We computed the statistic $f_{4}$ (Test, Mbuti; Altai, Denisovan) 511 in Fig. 2 on the HOIll dataset after merging with whole genome data on 3 Mbuti individuals 512 from Panel C of the Simons Genome Diversity Project ${ }^{49}$. We computed the dendrogram of

513 Extended Data Fig. 2 showing hierarchical clustering of populations with outgroup $f_{3}$ 514 statistics using the open source heatmap. 2 function of the gplots package in $R$.

\section{Negative correlation of Basal Eurasian ancestry with Neanderthal ancestry}

516 We used the $\mathrm{lm}$ function of $\mathrm{R}$ to fit a linear regression of the rate of allele sharing of a Test 517 population with the Altai Neanderthal as measured by $f_{4}$ (Test, Mbuti; Altai, Denisovan) as 518 the dependent variable, and the proportion of Basal Eurasian ancestry (Supplementary 519 Information, section 4) as the predictor variable,. Extrapolating from the fitted line, we obtain 520 the value of the statistic expected if Test is a population of $0 \%$ or $100 \%$ Basal Eurasian 521 ancestry. We then compute the ratio of the Neanderthal ancestry estimate in Basal Eurasians 
522 relative to non-Basal Eurasians as $f_{4}(100 \%$ Basal Eurasian, Mbuti; Altai, Denisovan $) / f_{4}(0 \%$

523 Basal Eurasian, Mbuti; Altai, Denisovan). We use a block jackknife ${ }^{48}$, dropping one of 100

524 contiguous blocks of the genome at a time, to estimate the value and standard error of this

525 quantity $(9 \pm 26 \%)$. We compute a $95 \%$ confidence interval based on the point estimate \pm 1.96 -

526 times the standard error: -42 to $60 \%$. We truncated to $0-60 \%$ on the assumption that Basal

527 Eurasians had no less Neanderthal admixture than Mbuti from sub-Saharan Africa.

\section{Estimation of $\mathbf{F}_{\text {ST }}$ coefficients}

529 We estimated $\mathrm{F}_{\mathrm{ST}}$ in smartpca ${ }^{17}$ with default parameters, inbreed: YES, and fstonly: YES.

\section{Admixture Graph modeling}

531 We carried out Admixture Graph modeling with the qpGraph software ${ }^{16}$ using Mbuti as an 532 outgroup unless otherwise specified.

\section{Testing for the number of streams of ancestry}

534 We used the qpWave $e^{35,50}$ software, described in Supplementary Information, section 10 of 535 ref. ${ }^{7}$, to test whether a set of 'Left' populations is consistent with being related via as few as $536 N$ streams of ancestry to a set of 'Right' populations by studying statistics of the form $X(u, v)$ $537=F_{4}\left(u_{0}, u ; v_{0}, v\right)$ where $u_{0}, v_{0}$ are basis populations chosen from the 'Left' and 'Right' sets 538 and $u, v$ are other populations from these sets. We use a Hotelling's $\mathrm{T}^{2}$ test $^{50}$ to evaluate 539 whether the matrix of size $(\mathrm{L}-1) *(\mathrm{R}-1)$, where L, R are the sizes of the 'Left' and 'Right' sets 540 has rank $m$. If this is the case, we can conclude that the 'Left' set is related via at least $N=m+1$ 541 streams of ancestry related differently to the 'Right' set.

\section{Inferring mixture proportions without an explicit phylogeny}

543 We used the qpAdm methodology described in Supplementary Information, section 10 of ref.

$544{ }^{7}$ to estimate the proportions of ancestry in a Test population deriving from a mixture of $N$

545 'reference' populations by exploiting (but not explicitly modeling) shared genetic drift with a 546 set of 'Outgroup' populations (Supplementary Information, section 7). We set the details:

547 YES parameter, which reports a normally distributed Z-score estimated with a block 548 jackknife for the difference between the statistics $f_{4}\left(u_{0}\right.$, Test $\left.; v_{0}, v\right)$ and $f_{4}\left(u_{0}\right.$, Estimated Test; $\left.549 v_{0}, v\right)$ where Estimated Test is $\sum_{i=1}^{N} \alpha_{i} f_{4}\left(u_{0}, R e f_{i} ; v_{0}, v\right)$, the average of these $f_{4}$-statistics 550 weighed by the mixture proportions $\alpha_{\mathrm{i}}$ from the $N$ reference populations. 


\section{Modeling admixture from ghost populations}

552 We model admixture from a 'ghost' (unobserved) population $X$ in the specific case that $X$ has

553 part of its ancestry from two unobserved ancestral populations $p$ and $q$. Any population $X$

554 composed of the same populations $p$ and $q$ resides on a line defined by two observed

555 reference populations $r_{1}$ and $r_{2}$ composed of the same elements $p$ and $q$ according to a

556 parametric equation $x=r_{1}+\lambda\left(r_{2}-r_{1}\right)$ with real-valued parameter $\lambda$. We define and solve

557 the optimization problem of fitting $\lambda$ and obtain mixture proportions (Supplementary

558 Information, section 10). 


\section{Figures Legends}

560 Figure 1: Genetic structure of ancient West Eurasia. (a) Sampling locations and times in six West

561 Eurasian regions. Sample sizes for each population are given below each bar. Abbreviations used: E:

562 Early, M: Middle, L: Late, HG: Hunter-Gatherer, N: Neolithic, ChL: Chalcolithic, BA: Bronze Age,

563 IA: Iron Age. (b) Principal components analysis of 991 present-day West Eurasians (grey points) with

564278 projected ancient samples (excluding the Upper Paleolithic Ust_Ishim, Kostenki14, and MA1).

565 To avoid visual clutter, population labels of present-day individuals are shown in Extended Data Fig.

566 1. (c) ADMIXTURE model-based clustering analysis of 2,583 present-day humans and 281 ancient

567 samples; we show the results only for ancient samples for $\mathrm{K}=11$ clusters.

568 Figure 2: Basal Eurasian ancestry explains the reduced Neanderthal admixture in West

569 Eurasians. Basal Eurasian ancestry estimates are negatively correlated to a statistic measuring

570 Neanderthal ancestry $f_{4}$ (Test, Mbuti; Altai, Denisovan).

571 Figure 3: Genetic differentiation and its dramatic decrease over time in West Eurasia. (a)

572 Pairwise $\mathrm{F}_{\mathrm{ST}}$ between 19 Ancient West Eurasian populations (arranged in approximate chronological

573 order), and select present-day populations. (b) Pairwise $\mathrm{F}_{\mathrm{ST}}$ distribution among populations belonging

574 to four successive time slices in West Eurasia; the median (red) and range of $\mathrm{F}_{\mathrm{ST}}$ is shown.

575 Figure 4: Modelling ancient West Eurasians, East Africans, East Eurasians and South Asians.

576 (a) All the ancient populations can be modelled as mixtures of two or three other populations and up 577 to four proximate sources (marked in colour). Mixture proportions inferred by $q p A d m$ are indicated by

578 the incoming arrows to each population. Clouds represent sets of more than one population. Multiple

579 admixture solutions are consistent with the data for some populations, and while only one solution is

580 shown here, Supplementary Information, section 7 presents the others. (b) A flat representation of the

581 graph showing mixture proportions from the four proximate sources. 


\section{Extended Data Tables and Extended Data Figure Legends}

583 Extended Data Table 1: No evidence for admixture related to sub-Saharan Africans in

584 Natufians. We computed the statistic $f_{4}$ (Natufian, Other Ancient; African, Chimp) varying African to

585 be Mbuti, Yoruba, Ju_hoan_North, or the ancient Mota individual. Gene flow between Natufians and

586 African populations would be expected to bias these statistics positive. However, we find most of

587 them to be negative in sign and all of them to be non-significant $(|Z|<3)$, providing no evidence that

588 Natufians differ from other ancient samples with respect to African populations.

\begin{tabular}{|llrrr|}
\hline Other Ancient & African & f $_{4}$ (Natufian, Other Ancient; African, Chimp) & Z & Number of SNPs \\
EHG & Mbuti & -0.00044 & -1.0 & 254033 \\
EHG & Yoruba & 0.00029 & 0.7 & 254033 \\
EHG & Ju_hoan_North & -0.00015 & -0.4 & 254033 \\
EHG & Mota & -0.00022 & -0.4 & 253986 \\
WHG & Mbuti & -0.00067 & -1.7 & 261514 \\
WHG & Yoruba & -0.00045 & -1.1 & 261514 \\
WHG & Ju_hoan_North & -0.00046 & -1.2 & 261514 \\
WHG & Mota & -0.00129 & -2.3 & 261461 \\
SHG & Mbuti & -0.00076 & -2.0 & 255686 \\
SHG & Yoruba & -0.00039 & -1.0 & 255686 \\
SHG & Ju_hoan_North & -0.00052 & -1.4 & 255686 \\
SHG & Mota & -0.00091 & -1.7 & 255641 \\
Switzerland_HG & Mbuti & -0.00018 & -0.4 & 261322 \\
Switzerland_HG & Yoruba & 0.00019 & 0.4 & 261322 \\
Switzerland_HG & Ju_hoan_North & 0.00009 & 0.2 & 261322 \\
Switzerland_HG & Mota & -0.00062 & -0.9 & 261276 \\
Kostenki14 & Mbuti & 0.00034 & 0.7 & 246765 \\
Kostenki14 & Yoruba & 0.00120 & 2.3 & 246765 \\
Kostenki14 & Ju_hoan_North & 0.00069 & 1.4 & 246765 \\
Kostenki14 & Mota & 0.00036 & 0.5 & 246719 \\
MA1 & Mbuti & -0.00038 & -0.7 & 191819 \\
MA1 & Yoruba & 0.00009 & 0.2 & 191819 \\
MA1 & Ju_hoan_North & -0.00010 & -0.2 & 191819 \\
MA1 & Mota & -0.00038 & -0.5 & 191782 \\
CHG & Mbuti & -0.00051 & -1.2 & 261505 \\
CHG & Yoruba & -0.00012 & -0.3 & 261505 \\
CHG & Ju_hoan_North & -0.00013 & -0.3 & 261505 \\
CHG & Mota & -0.00042 & -0.7 & 261456 \\
Iran_N & Mbuti & -0.00018 & -0.4 & 232927 \\
Iran_N & Yoruba & 0.00036 & 0.8 & 232927 \\
Iran_N & Ju_hoan_North & 0.00041 & 0.9 & 232927 \\
Iran_N & Mota & 0.00006 & 0.1 & 232880 \\
\hline
\end{tabular}


bioRxiv preprint doi: https://doi.org/10.1101/059311; this version posted June 16, 2016. The copyright holder for this preprint (which was not

591 Extended Data Table 2: Admixture $\boldsymbol{f}_{3}$-statistics. We show the lowest Z-score of the statistic $f_{3}($ Test;

592 Reference ${ }_{1}$, Refrence $_{2}$ ) for every ancient Test population with at least 2 individuals and every pair

593 (Reference ${ }_{1}$, Refrence $_{2}$ ) of ancient or present-day source populations. Z-scores lower than -3 are

594 highlighted and indicate that the Test population is admixed from sources related to (but not identical

595 to) the reference populations. Z-scores greater than -3 are consistent with the population either being

596 admixed or not.

\begin{tabular}{|c|c|c|c|c|c|}
\hline Test & Reference $_{1}$ & Reference $_{2}$ & $f_{3}\left(\right.$ Test; Reference $_{1}$, Refrence $\left._{2}\right)$ & Z-score & Number of SNPs \\
\hline Anatolia_N & Iberia_BA & Levant_N & -0.00034 & -0.2 & 111632 \\
\hline Armenia_ChL & $\mathrm{EHG}^{-}$ & Levant_N $\mathrm{N}$ & -0.00249 & -1.5 & 167020 \\
\hline Armenia_EBA & Anatolia_N & $\mathrm{CHG}$ & -0.01017 & -7.9 & 195596 \\
\hline Armenia_MLBA & Anatolia_N & Steppe_EMBA & -0.00809 & -7.3 & 203796 \\
\hline $\mathrm{CHG}$ & Anatolia_ChL & Iran_Hotullıb & 0.02612 & 3.6 & 9884 \\
\hline EHG & Steppe_Eneolithic & Switzerland_HG & -0.00282 & -0.9 & 67938 \\
\hline Europe_EN & Anatolia_N $\mathrm{N}$ & WHG & -0.00494 & -11.2 & 380684 \\
\hline Europe_LNBA & Europe_MNChL & Steppe_EMBA & -0.00920 & -41.8 & 414782 \\
\hline Europe_MNChL & Anatolia_N & WHG & -0.01351 & -26.8 & 363672 \\
\hline Iran_Ch̄ & Anatolia_N & Iran_N & -0.01285 & -10.6 & 167941 \\
\hline Iran_N & Iran_LN ${ }^{-}$ & Ganà & -0.00462 & -1.1 & 17804 \\
\hline Levānt_BA & Iran_N & Levant_N & -0.00853 & -4.7 & 118269 \\
\hline Levant_N & Europe_MNChL & Natufian & -0.00671 & -3.6 & 61845 \\
\hline Natufian & Iberia_B̄A & Iran_HotullIb & 0.07613 & 3.4 & 1054 \\
\hline SHG & Steppē_Eneolithic & Switz̄erland_HG & 0.00728 & 3.2 & 154825 \\
\hline Steppe_EMBA & $\mathrm{EHG}-\mathrm{Ha}$ & Abkhasian & -0.00756 & -11.2 & 349359 \\
\hline Steppe_Eneolithic & EHG & Iran_LN & -0.01637 & -4.2 & 25100 \\
\hline Steppe_MLBA & Europe_MNChL & Steppe_EMBA & -0.00573 & -18.0 & 378298 \\
\hline WHG ${ }^{-}$ & Switzerland_HG & Saudi ${ }^{-}$ & -0.01562 & -7.7 & 218758 \\
\hline Abkhasian & $\mathrm{CHG}$ & Sardinian & -0.00754 & -13.1 & 387956 \\
\hline Adygei & Anatolia_N & Eskimo & -0.00699 & -14.4 & 413128 \\
\hline Albanian & Europe_EN & Burusho & -0.00650 & -16.8 & 395851 \\
\hline Armenian & Anatolia_N & Sindhi & -0.00603 & -19.5 & 406021 \\
\hline Assyrian & Iran_N & Sardinian & -0.00672 & -11.8 & 309055 \\
\hline Balkar & Anatolia_N & Chukchi & -0.00975 & -18.8 & 401928 \\
\hline Basque & Switzerland_HG & Druze & -0.00726 & -12.6 & 416070 \\
\hline BedouinA & Europe_EN & Yoruba & -0.01584 & -42.8 & 460762 \\
\hline BedouinB & Iran_Hotulllib & Natufian & 0.01384 & 4.1 & 32266 \\
\hline Belarusian & $W H \bar{G}$ & Iranian & -0.00974 & -19.8 & 392363 \\
\hline Bulgarian & Anatolia_N & Steppe_EMBA & -0.00807 & -26.7 & 400263 \\
\hline Canary_Islander & Europe_MNChL & Mende & -0.00829 & -5.9 & 353172 \\
\hline Chechen & Anatolia_N & Eskimo & -0.00440 & -7.9 & 396678 \\
\hline Croatian & WHG & Druze & -0.00871 & -18.6 & 394032 \\
\hline Cypriot & Anatolia_N & Sindhi & -0.00562 & -16.1 & 401141 \\
\hline Czech & SHG & Druze & -0.00919 & -21.7 & 374705 \\
\hline Druze & Iran_N & Sardinian & -0.00269 & -5.8 & 343813 \\
\hline English & Steppe_EMBA & Sardinian & -0.00628 & -20.6 & 402502 \\
\hline Estonian & $\mathrm{SHG}$ & Druze & -0.00789 & -17.6 & 371575 \\
\hline Finnish & SHG & Assyrian & -0.00716 & -12.6 & 355744 \\
\hline French & Steppe_EMBA & Sardinian & -0.00669 & -37.9 & 441807 \\
\hline Georgian & $\mathrm{CHG}$ & Sardinian & -0.00782 & -13.7 & 390744 \\
\hline German & WHG & Druze & -0.01103 & -22.9 & 391302 \\
\hline Greek & Europe_EN & Pathan & -0.00600 & -30.0 & 421984 \\
\hline Hungarian & Steppe_EMBA & Sardinian & -0.00644 & -31.2 & 420017 \\
\hline Icelandic & WHG & Abkhasian & -0.00974 & -17.0 & 394625 \\
\hline Iranian & Anatolia_N & Sindhi & -0.00594 & -30.9 & 443011 \\
\hline Irish & Steppe_EMBA & Sardinian & -0.00590 & -22.8 & 416663 \\
\hline Irish_Ulster & $\mathrm{SHG}$ & Assyrian & -0.00909 & -15.6 & 350547 \\
\hline Italian_North & Europe_EN & Steppe_EMBA & -0.00627 & -26.4 & 419169 \\
\hline Italian_South & Iberia_BA & Iran_Hotulllb & 0.01224 & 2.6 & 17678 \\
\hline Jew_Asshkenazi & Anatolia_N & Koryak & -0.00532 & -9.4 & 389012 \\
\hline Jew_Georgian & Iran_N & Sardinian & -0.00306 & -4.2 & 292410 \\
\hline Jew_Iranian & Iran_N & Sardinian & -0.00385 & -5.8 & 302446 \\
\hline Jew_Iraqi & Iran_N & Sardinian & -0.00486 & -6.5 & 287673 \\
\hline Jew_Libyan & Europe_EN & Yoruba & -0.00397 & -7.2 & 415797 \\
\hline Jew_Moroccan & Europe_EN & Yoruba & -0.00649 & -10.9 & 405193 \\
\hline Jew_Tunisian & Anatolia_N & Mende & -0.00276 & -4.1 & 399354 \\
\hline Jew_Turkish & Anatolia_N & Burusho & -0.00571 & -16.4 & 405254 \\
\hline Jew_Yemenite & Natufian & Kalash & -0.00341 & -3.8 & 174052 \\
\hline Jordānian & Europe_EN & Yoruba & -0.01283 & -26.7 & 423649 \\
\hline Kumyk & Anatolia_N & Chukchi & -0.01025 & -19.6 & 396439 \\
\hline Lebanese & Anatolia_N & Yoruba & -0.01022 & -19.5 & 414854 \\
\hline Lebanese_Christian & Anatolia_N & Sindhi & -0.00504 & -15.7 & 404858 \\
\hline Lebanese_Muslim & Anatolia_N & Brahmin_Tiwari & -0.00616 & -20.4 & 415129 \\
\hline Lezgin - & Steppe_EMBA & Jew_Yemenite & -0.00481 & -13.1 & 398974 \\
\hline Lithuanian & WHG & Abkhasian & -0.00999 & -17.7 & 386718 \\
\hline Maltese & Anatolia_N & Brahmin_Tiwari & -0.00518 & -14.5 & 404438 \\
\hline Mordovian & WHG & Iranian & -0.00912 & -18.4 & 395230 \\
\hline North_Ossetian & Anatolia_N & Chukchi & -0.00894 & -17.2 & 401729 \\
\hline Norwēgian & WHG - & Abkhasian & -0.00957 & -16.5 & 393546 \\
\hline Orcadian & SHG & Druze & -0.00662 & -15.8 & 379656 \\
\hline Palestinian & Europe_EN & Yoruba & -0.01129 & -31.3 & 464066 \\
\hline Polish & SHG & Druze & -0.00924 & -27.8 & 394654 \\
\hline Romanian & Europe_EN & Steppe_EMBA & -0.00549 & -16.9 & 397119 \\
\hline Russian & $\mathrm{SHG}$ & Turkish & -0.00731 & -25.0 & 398393 \\
\hline Sardinian & Anatolia_N & Switzerland_HG & -0.00587 & -9.6 & 417931 \\
\hline Saudi & Anatolia_N & Dinka & -0.00326 & -5.1 & 404923 \\
\hline Scottish & Steppe_EMBA & Sardinian & -0.00622 & -26.6 & 426660 \\
\hline Shetlandic & WHG ${ }^{-}$ & Abkhasian & -0.00868 & -14.6 & 386562 \\
\hline Sicilian & Anatolia_N & Brahmin_Tiwari & -0.00646 & -22.2 & 411481 \\
\hline Sorb & SHG & Palestinian & -0.00787 & -16.8 & 366924 \\
\hline Spanish & Steppe_EMBA & Sardinian & -0.00557 & -32.2 & 447735 \\
\hline Spanish_North & WHG & Armenian & -0.00825 & -10.9 & 356832 \\
\hline Syrian & Europe_EN & Dinka & -0.01002 & -17.3 & 410920 \\
\hline Turkish & Europe_EN & Sindhi & -0.00709 & -41.1 & 448975 \\
\hline Ukrainian & WHG & Abkhasian & -0.01183 & -21.4 & 388282 \\
\hline
\end{tabular}


597 Extended Data Figure 1: Principal components analysis of 991 present-day West Eurasians. The

598 PCA analysis is performed on the same set of individuals as are reported in Fig. 1b, using

599 EIGENSOFT. Here, we color the samples by population (to highlight the present-day populations)

600 instead of using grey points as in Fig. 1b (where the goal is to highlight ancient samples).

601 Extended Data Figure 2: Outgroup $\boldsymbol{f}_{3}(\mathbf{M b u t i} \mathbf{X}, \mathbf{Y})$ for pairs of ancient populations. The

602 dendrogram is plotted for convenience and should not be interpreted as a phylogenetic tree. Areas of

603 high shared genetic drift are 'yellow' and include from top-right to bottom-left along the diagonal:

604 early Anatolian and European farmers; European hunter-gatherers, Steppe populations and ones

605 admixed with steppe ancestry; populations from the Levant from the Epipaleolithic (Natufians) to the

606 Bronze Age; populations from Iran from the Mesolithic to the Late Neolithic.

607 Extended Data Figure 3: Reduction of genetic differentiation in West Eurasia over time. We

608 measure differentiation by $\mathrm{F}_{\mathrm{ST}}$. Each column of the $5 \times 5$ matrix of plots represents a major region and

609 each row the earliest population with at least two individuals from each major region.

Extended Data Figure 4: West Eurasian related admixture in East Africa, Eastern Eurasia and ancestry in different Eastern African population in the dataset of Pagani et al. (2012); the remainder of the ancestry is a clade with Mota, a 4,500 year old sample from Ethiopia. (c) EHG ancestry in Eastern Eurasians, or (d) Afontova Gora (AG2) ancestry in Eastern Eurasians; the remainder of their ancestry is a clade with Onge. (e) Mixture proportions for South Asian populations showing that they can be modelled as having West Eurasian-related ancestry similar to that in populations from both the Eurasian steppe and Iran.

Extended Data Figure 5: Inferred position of ancient populations in West Eurasian PCA according to the model of Fig. 4.

Extended Data Figure 6: Admixture from ghost populations using 'cline intersection'. We model each Test population (purple) in panels (a-f) as a mixture (pink) of a fixed reference population (blue) and a ghost population (orange) residing on the cline defined by two other populations (red and green) according to the visualization method of Supplementary Information, section 10. (a) Early/Middle Bronze Age steppe populations are a mixture of Iran_ChL and a population on the $\mathrm{WHG} \rightarrow \mathrm{SHG}$ cline. (b) Scandinavian hunter-gatherers (SHG) are a mixture of WHG and a population on the Iran_ChL $\rightarrow$ Steppe_EMBA cline. (c) Caucasus hunter-gatherers (CHG) are a mixture of Iran_N and both WHG and EHG. (d) Late Neolithic/Bronze Age Europeans are a mixture of the preceding Europe_MNChL population and a population with both EHG and Iran_ChL ancestry. (e) Somali are a mixture of Mota and a population on the Iran_ChL $\rightarrow$ Levant_BA cline. (f) Eastern European huntergatherers (EHG) are a mixture of WHG and a population on the Onge $\rightarrow$ Han cline. 
631 Extended Data Figure 7: Admixture from a 'ghost' ANE population into both European and

632 Eastern Eurasian ancestry. EHG, and Upper Paleolithic Siberians Mal'ta 1 (MA1) and Afontova

633 Gora 2 (AG2) are positioned near the intersection of clines formed by European hunter-gatherers

634 (WHG, SHG, EHG) and Eastern non-Africans in the space of outgroup $f_{3}$-statistics of the form

$635 f_{3}$ (Mbuti; Papuan, Test) and $f_{3}$ (Mbuti; Switzerland_HG, Test).

\section{References}

638 1. Barker, G. \& Goucher, C. The Cambridge World History Volume II: A world with agriculture, 12,000 BCE-500 CE. (Cambridge University Press, 2015).

2. Cavalli-Sforza, L. L. \& Paolo, M. The History and Geography of Human Genes. (Princeton University Press, 1994).

3. Gamba, C. et al. Genome flux and stasis in a five millennium transect of European prehistory. Nat. Commun. 5, 5257 (2014).

4. Pinhasi, R. et al. Optimal Ancient DNA Yields from the Inner Ear Part of the Human Petrous Bone. PLoS ONE 10, e0129102, (2015).

5. $\mathrm{Fu}$, Q. et al. DNA analysis of an early modern human from Tianyuan Cave, China. Proc.

Natl. Acad. Sci. USA 110, 2223-2227, (2013).

6. Fu, Q. et al. An early modern human from Romania with a recent Neanderthal ancestor.

Nature 524, 216-+, (2015).

7. Haak, W. et al. Massive migration from the steppe was a source for Indo-European languages in Europe. Nature 522, 207-211, (2015).

8. Mathieson, I. et al. Genome-wide patterns of selection in 230 ancient Eurasians. Nature 528, 499-503, (2015).

9. Jones, E. R. et al. Upper Palaeolithic genomes reveal deep roots of modern Eurasians. Nat. Commun. 6, 8912, (2015).

10. Allentoft, M. E. et al. Population genomics of Bronze Age Eurasia. Nature 522, 167-172, (2015).

11. Fu, Q. et al. Genome sequence of a 45,000-year-old modern human from western Siberia. Nature 514, 445-449, (2014).

12. Günther, T. et al. Ancient genomes link early farmers from Atapuerca in Spain to modern-day Basques. Proceedings of the National Academy of Sciences 112, 11917-11922, (2015).

13. Lazaridis, I. et al. Ancient human genomes suggest three ancestral populations for presentday Europeans. Nature 513, 409-413, (2014).

14. Olalde, I. et al. A common genetic origin for early farmers from Mediterranean Cardial and Central European LBK cultures. Mol. Biol. Evol. 32, 3132-3142, (2015).

15. Raghavan, M. et al. Upper Palaeolithic Siberian genome reveals dual ancestry of Native Americans. Nature 505, 87-91, (2014).

16. Patterson, N. et al. Ancient admixture in human history. Genetics 192, 1065-1093, (2012).

17. Patterson, N., Price, A. L. \& Reich, D. Population structure and eigenanalysis. PLoS Genet. 2, e190, (2006).

18. Alexander, D. H., Novembre, J. \& Lange, K. Fast model-based estimation of ancestry in unrelated individuals. Genome Res. 19, 1655-1664, (2009).

19. Prufer, K. et al. The complete genome sequence of a Neanderthal from the Altai Mountains. Nature 505, 43-49, (2014).

20. Kim, Bernard Y. \& Lohmueller, Kirk E. Selection and Reduced Population Size Cannot Explain Higher Amounts of Neandertal Ancestry in East Asian than in European Human Populations. The American Journal of Human Genetics 96, 454-461, (2015).

21. Meyer, M. et al. A High-Coverage Genome Sequence from an Archaic Denisovan Individual. Science 338, 222-226, (2012). 
680 22. Vernot, B. \& Akey, Joshua M. Complex History of Admixture between Modern Humans and

23. Wall, J. D. et al. Higher Levels of Neanderthal Ancestry in East Asians Than in Europeans. Genetics 194, 199-209, (2013).

24. Green, R. E. et al. A draft sequence of the Neandertal genome. Science 328, 710-722, (2010).

25. Brace, C. L. et al. The questionable contribution of the Neolithic and the Bronze Age to European craniofacial form. Proc. Natl. Acad. Sci. U. S. A. 103, 242-247, (2006).

26. Ferembach, D. Squelettes du Natoufien d'Israel., etude anthropologique. . L'Anthropologie 65, 46-66, (1961).

27. Fadhlaoui-Zid, K. et al. Genome-Wide and Paternal Diversity Reveal a Recent Origin of Human Populations in North Africa. PLoS ONE 8, e80293, (2013).

28. Henn, B. M. et al. Genomic ancestry of North Africans supports back-to-Africa migrations. PLoS genetics 8, e1002397, (2012).

29. Bhatia, G., Patterson, N., Sankararaman, S. \& Price, A. L. Estimating and interpreting F(ST): The impact of rare variants. Genome Res. 23, 1514-1521, (2013).

30. Fernandez, E. et al. Ancient DNA analysis of 8000 B.C. near eastern farmers supports an early neolithic pioneer maritime colonization of Mainland Europe through Cyprus and the Aegean Islands. PLoS genetics 10, e1004401, (2014).

31. Ammerman, A. J., Pinhasi, R. \& Banffy, E. Comment on "Ancient DNA from the first European farmers in 7500-year-old Neolithic sites". Science 312, 1875; author reply 1875 , (2006).

32. Pagani, L. et al. Ethiopian Genetic Diversity Reveals Linguistic Stratification and Complex Influences on the Ethiopian Gene Pool. Am. J. Hum. Genet. 91, 83-96, (2012).

33. Pickrell, J. K. et al. Ancient west Eurasian ancestry in southern and eastern Africa. Proc. Natl. Acad. Sci. USA 111, 2632-2637, (2014).

34. Keller, A. et al. New insights into the Tyrolean Iceman's origin and phenotype as inferred by whole-genome sequencing. Nat. Commun. 3, 698, (2012).

35. Moorjani, P. et al. Genetic evidence for recent population mixture in India. Am. J. Hum. Genet. 93, 422-438, (2013).

36. Reich, D., Thangaraj, K., Patterson, N., Price, A. L. \& Singh, L. Reconstructing Indian population history. Nature 461, 489-494, (2009).

37. Fu, Q. et al. The genetic history of Ice Age Europe. Nature 534, 200-205, (2016).

38. Dabney, J. et al. Complete mitochondrial genome sequence of a Middle Pleistocene cave bear reconstructed from ultrashort DNA fragments. Proceedings of the National Academy of Sciences of the United States of America 110, 15758-15763, (2013).

39. Rohland, N., Harney, E., Mallick, S., Nordenfelt, S. \& Reich, D. Partial uracil-DNAglycosylase treatment for screening of ancient DNA. Philosophical Transactions of the Royal Society of London B: Biological Sciences 370, DOI: 10.1098/rstb.2013.0624 (2014).

40. Briggs, A. W. et al. Removal of deaminated cytosines and detection of in vivo methylation in ancient DNA. Nucleic acids research 38, e87, (2010).

41. Korlevic, P. et al. Reducing microbial and human contamination in DNA extractions from ancient bones and teeth. BioTechniques 59, 87-93, (2015).

42. Meyer, M. et al. A mitochondrial genome sequence of a hominin from Sima de los Huesos. Nature 505, 403-406, (2014).

43. Behar, D. M. et al. A “Copernican” Reassessment of the Human Mitochondrial DNA Tree from its Root. Am. J. Hum. Genet. 90, 675-684, (2012).

44. Li, H. \& Durbin, R. Fast and accurate short read alignment with Burrows-Wheeler transform. Bioinformatics 25, 1754-1760, (2009).

45. Korneliussen, T. S., Albrechtsen, A. \& Nielsen, R. ANGSD: Analysis of Next Generation Sequencing Data. BMC Bioinformatics 15, 1-13, (2014).

46. Purcell, S. et al. PLINK: a tool set for whole-genome association and population-based linkage analyses. Am. J. Hum. Genet. 81, 559-575, (2007).

47. Chang, C. et al. Second-generation PLINK: rising to the challenge of larger and richer datasets. GigaScience 4, 7, (2015). 
734 48. Busing, F. T. A., Meijer, E. \& Leeden, R. Delete-m Jackknife for Unequal m. Statistics and

49. Sudmant, P. H. et al. Global diversity, population stratification, and selection of human copynumber variation. Science 349, (2015).

740

50. Reich, D. et al. Reconstructing Native American population history. Nature 488, 370-374, (2012). 
bioRxiv preprint doi: https://doi.org/10.1101/059311; this version posted June 16, 2016. The copyright holder for this preprint (which was not certified by peer review) is the author/funder, who has granted bioRxiv a license to display the preprint in perpetuity. It is made available under aCC-BY-NC-ND 4.0 International license.

a

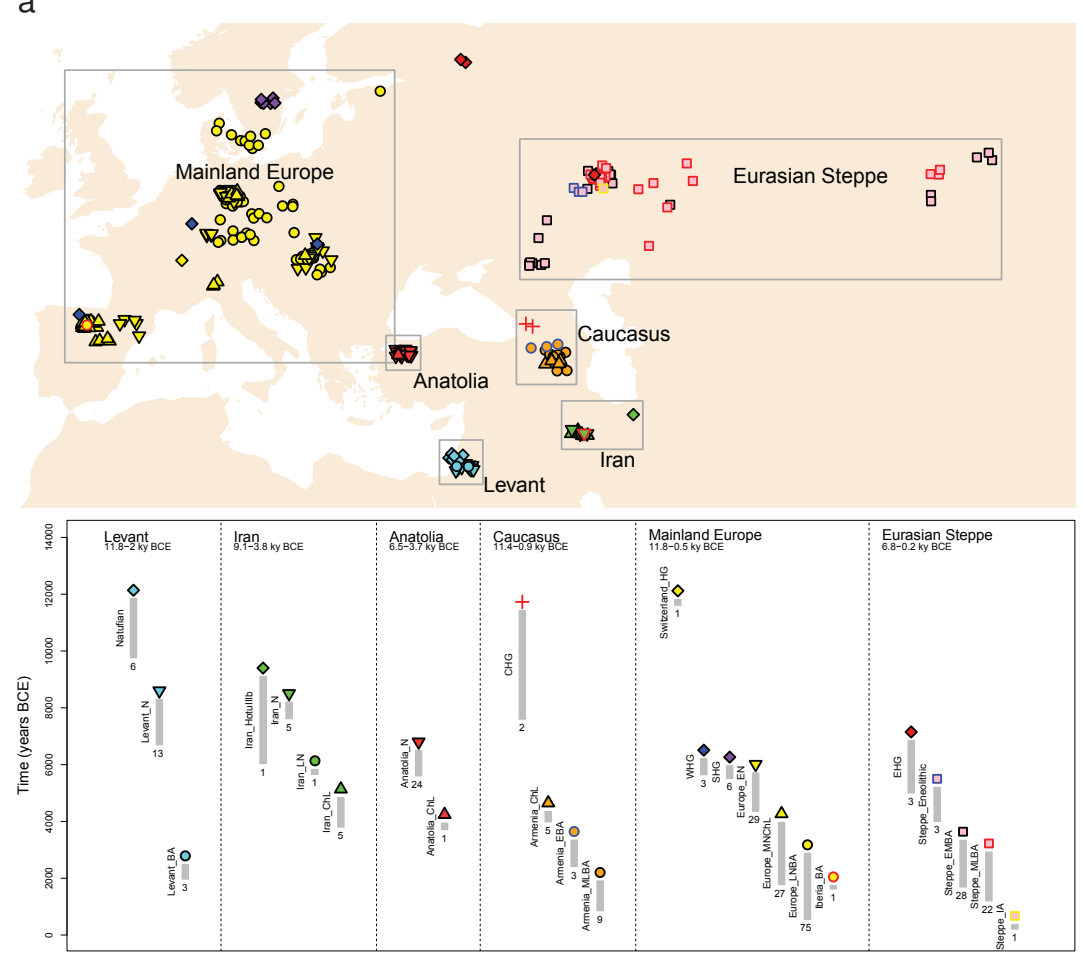

b

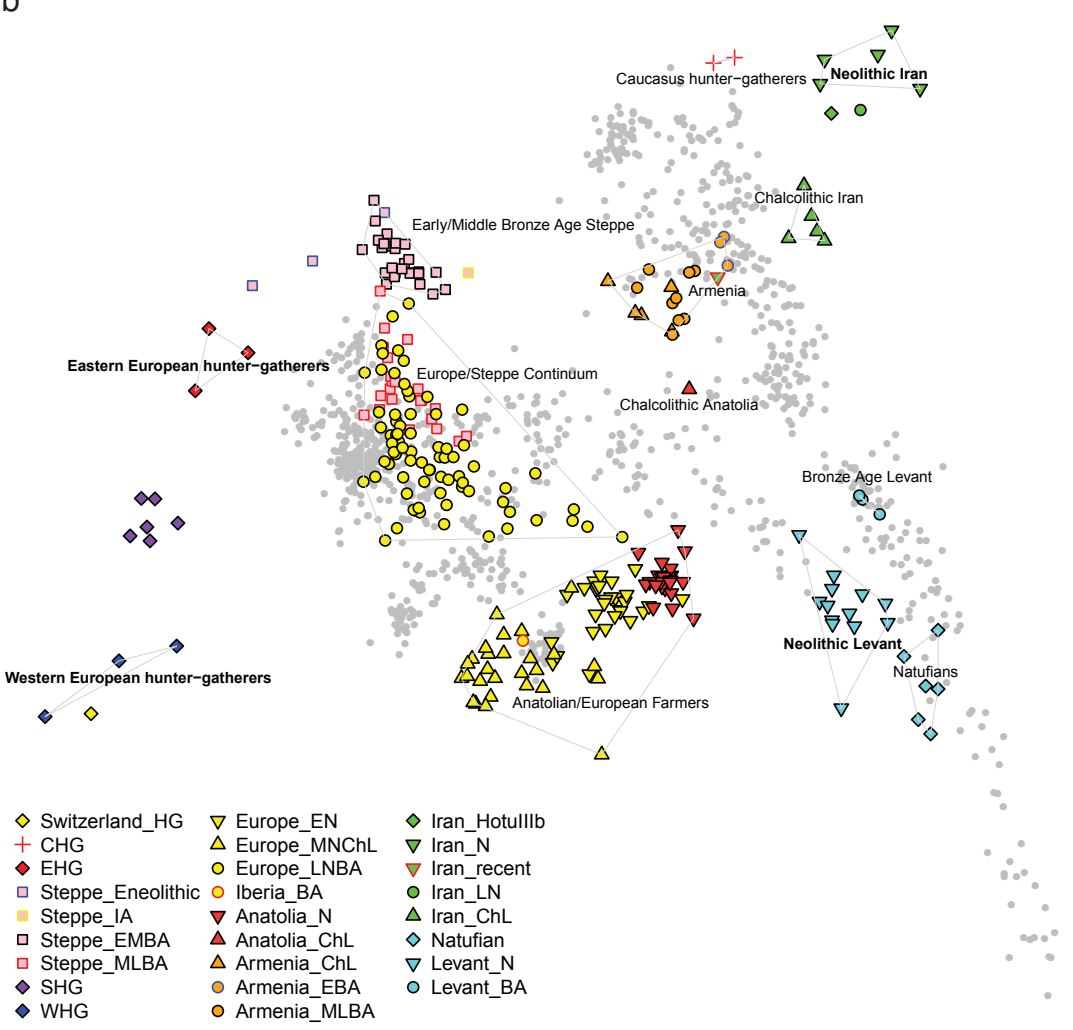

C
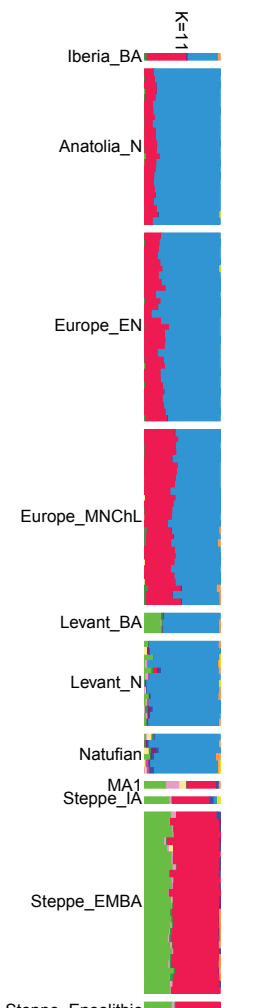

Steppe_Eneolithic

EHGIL

WHG

SHG

Switzerland $H G$
Kostenki14

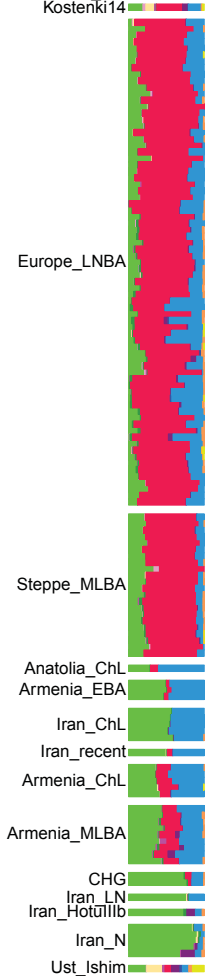

Figure 1 
bioRxiv preprint doi: https://doi.org/10.1101/059311; this version posted June 16, 2016. The copyright holder for this preprint (which was not certified by peer review) is the author/funder, who has granted bioRxiv a license to display the preprint in perpetuity. It is made available under aCC-BY-NC-ND 4.0 International license.
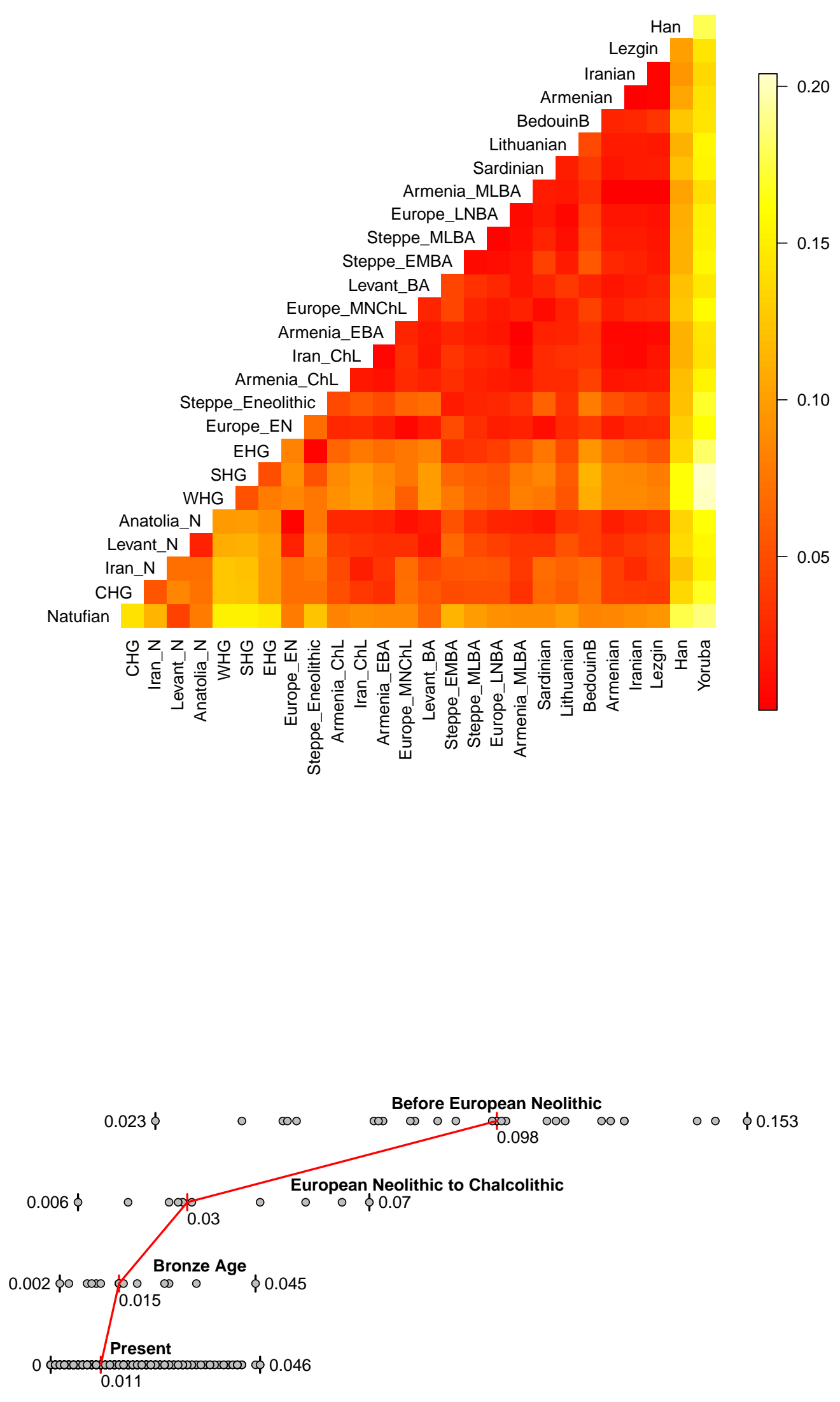
$\sim 0.6$

$\sim 0.9$

bioRxiv prêprint doi: https://doi.org/10.1101/059311; thistersion posted June 16, 2016. The ${ }^{1}$ copyright holder for this preprint (which was not certified by beer review) is thenaldthor/funder, who has granted diaiRxir-aticenseto display the preprint in perpetuity. It is made available under

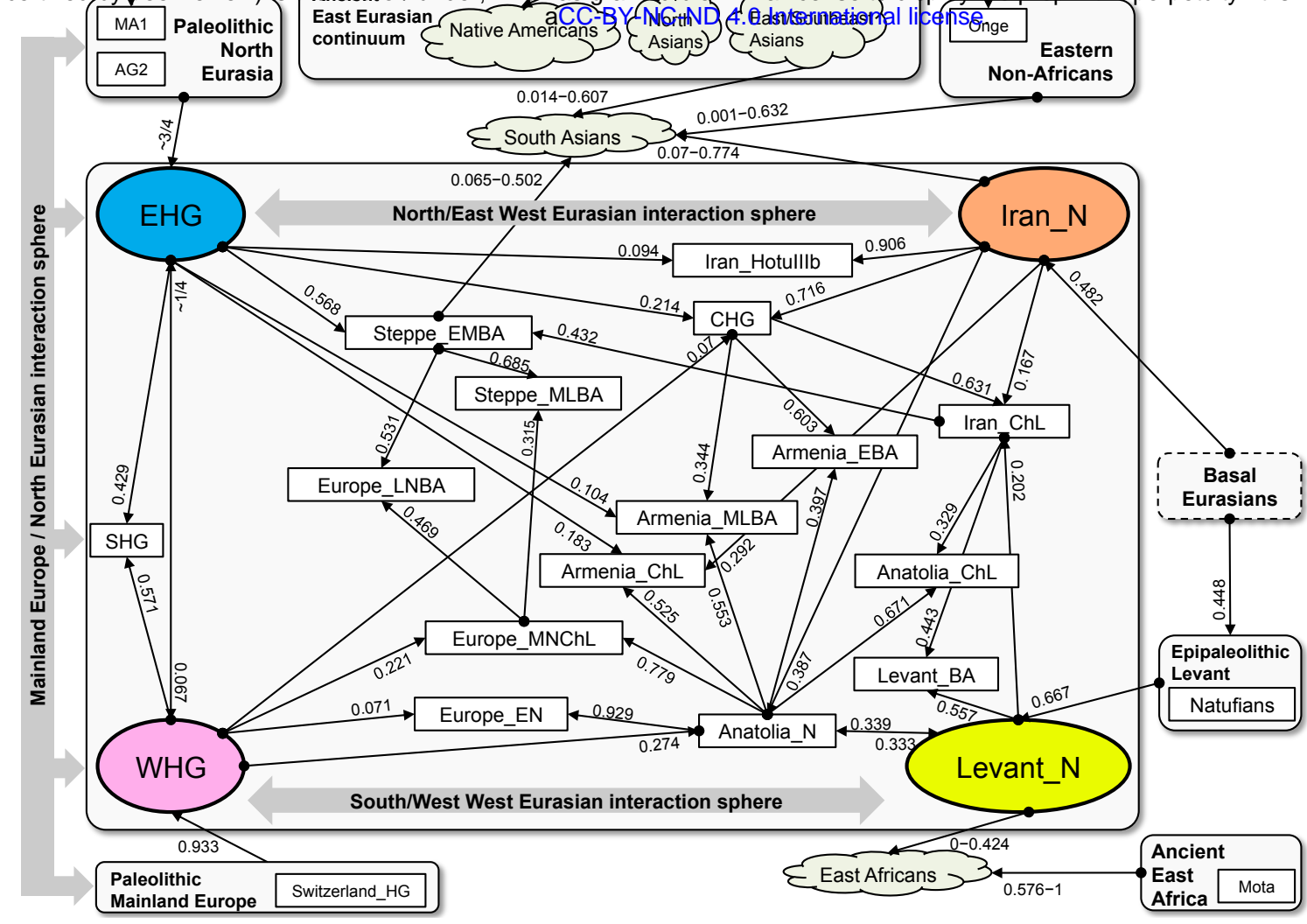

b

$\mathrm{CHG}$

Armenia_ChL

Armenia_EBA

Armenia_MLBA

Iran_Hotulllb

Iran_N

Iran_ChL

Levant_N

Levant_BA

Anatolia_N

Anatolia_ChL

WHG

SHG

Europe_EN

Europe_MNChL

Europe_LNBA

EHG

Steppe_EMBA

Steppe_MLBA

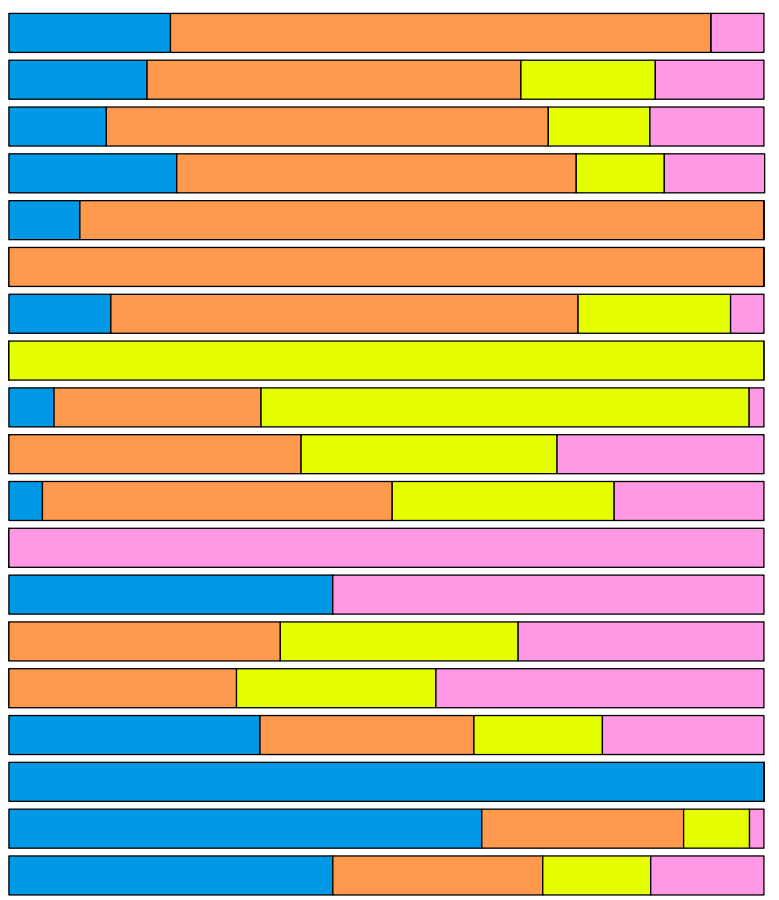

Figure 4 


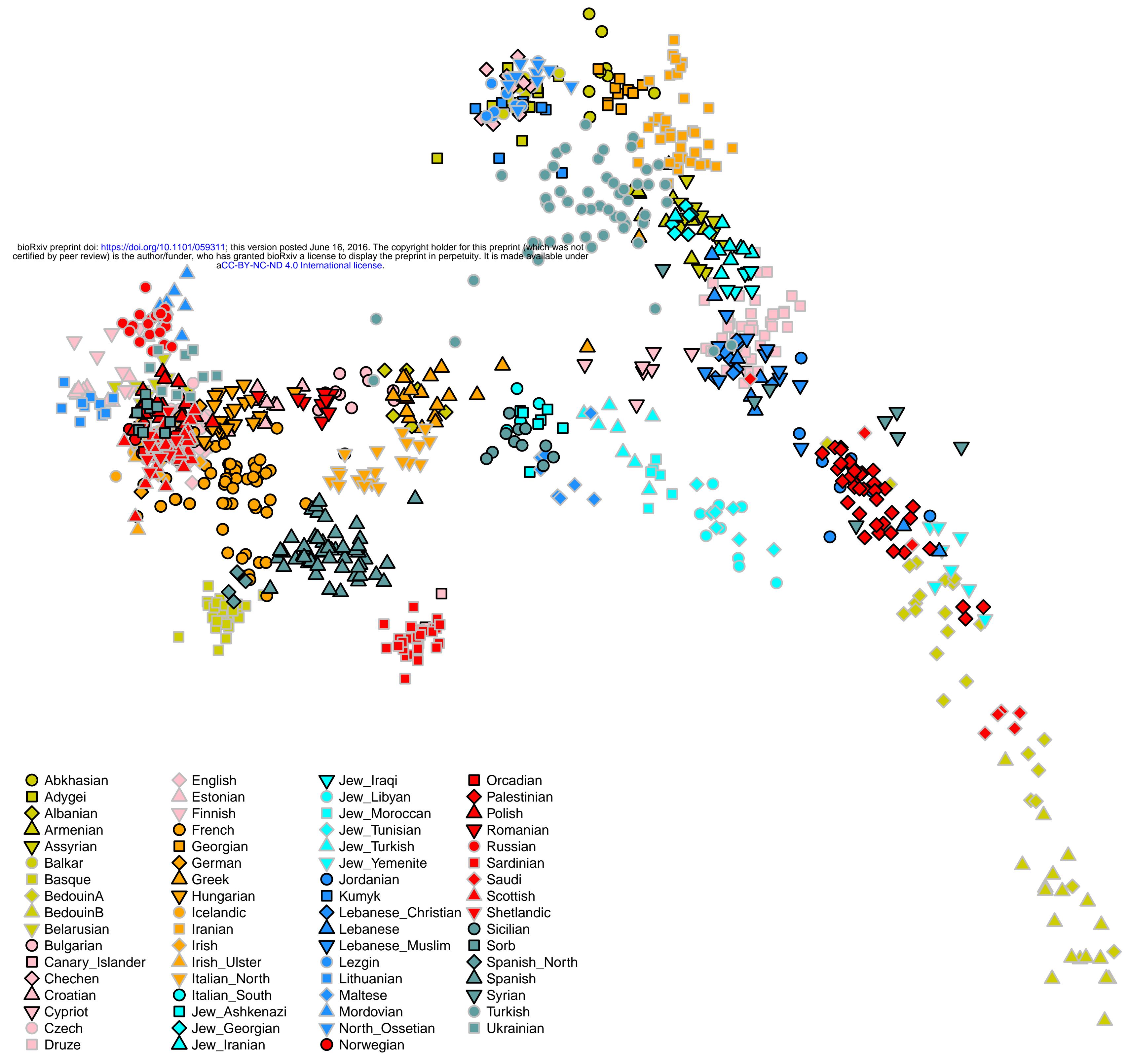


WHG

WHG

WHG

WHG

bioß̊̊xiv preprint doi: https://doi.org/10.1101/059311; this version posted June 16, 2016. The copyright holder for this preprint (which was not

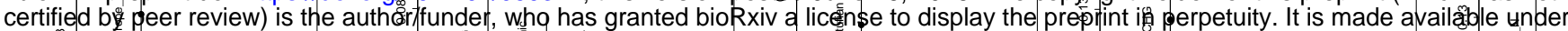

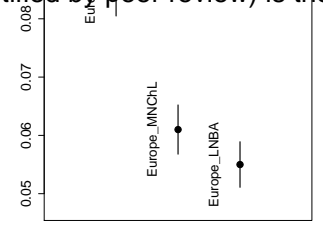

Mainland Europe

EHG
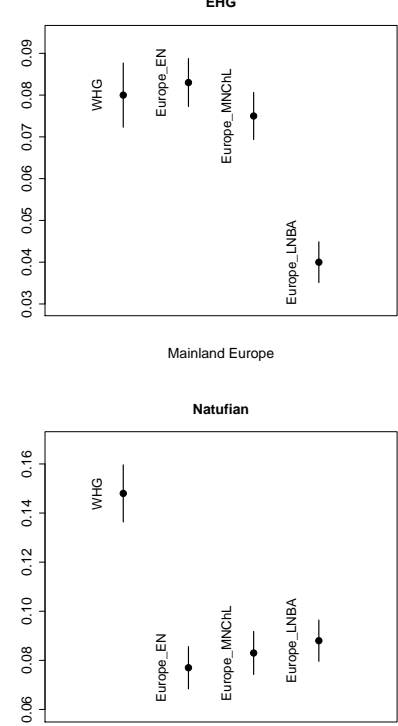

Mainland Europe

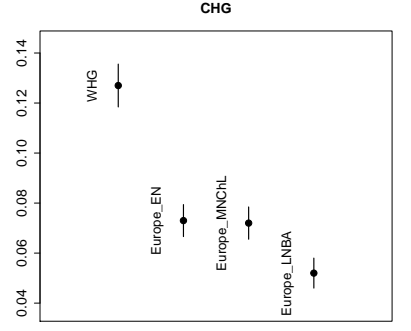

Mainland Europe

Iran $\mathbf{N}$

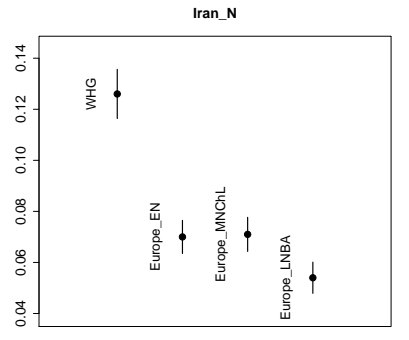

Mainland Europe

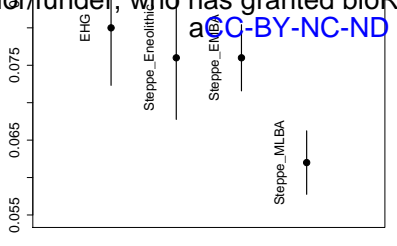

Eurasian steppe
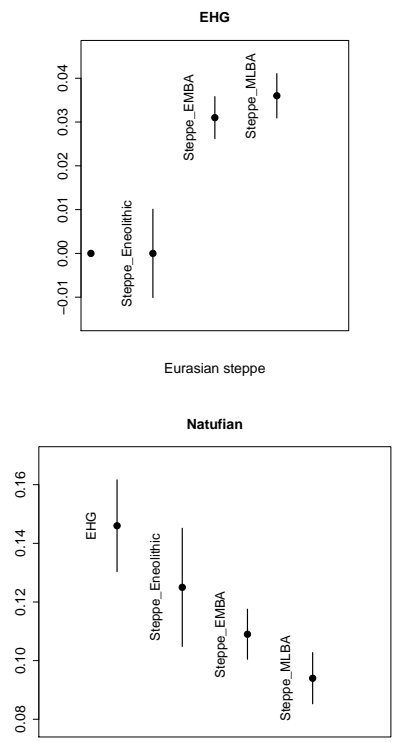

CHG

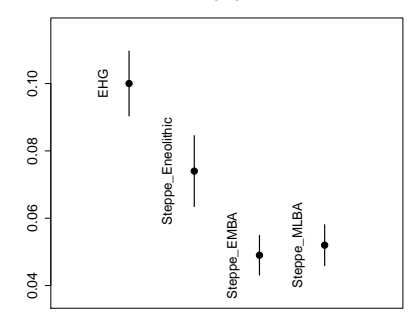

Eurasian steppe

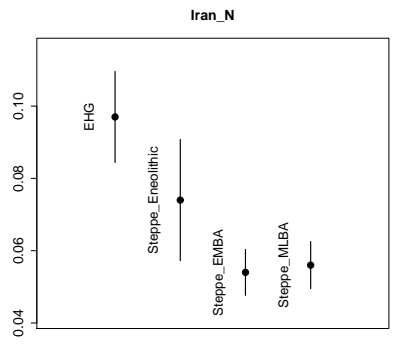

Eurasian steppe

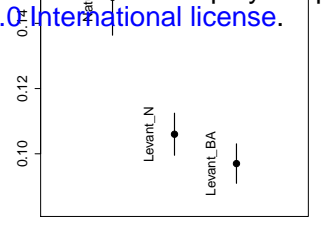

Levant

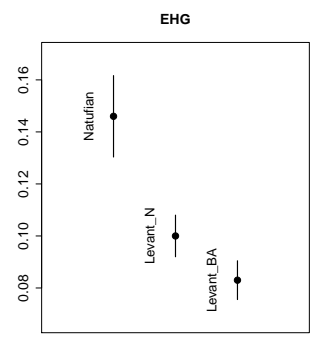

evant

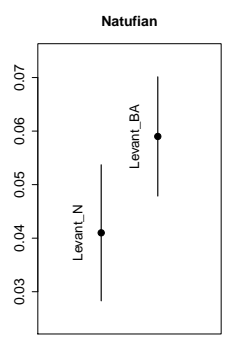

Levant

$\mathrm{CHO}$

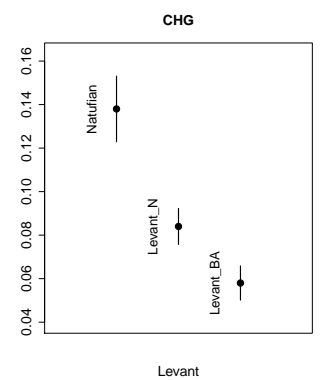

Iran_N

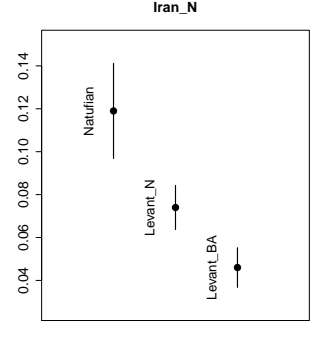

Levant

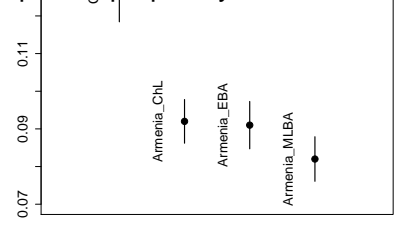

EHG
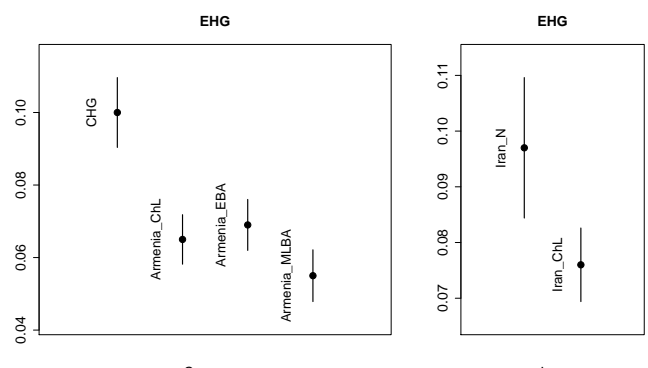

ran
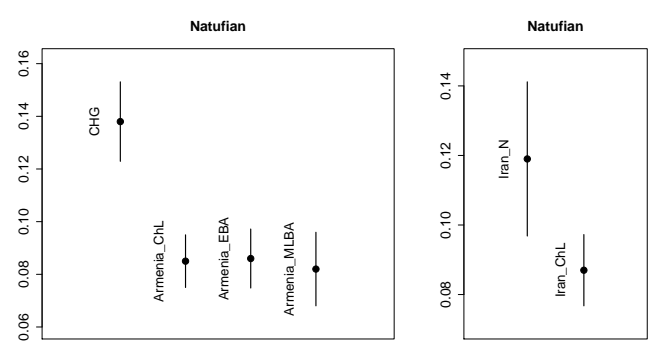

Iran
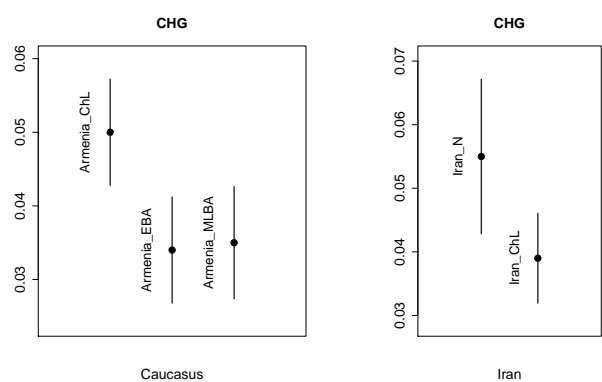

Iran

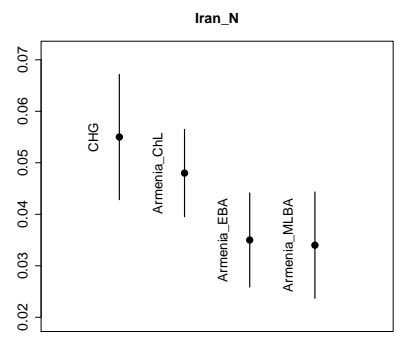

Caucasus

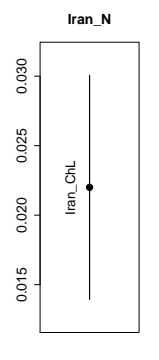

Extended Data Figure 3 
bioRxiv preprint doi: https:I//doi. Org /10.1101/059311; this version posted June 16, 2016. The copyright holder for this preprint (which was not certified by peer review) is the author/funder, who has granted bioRxiv a license to display the preprint in perpetuity. It is made available under
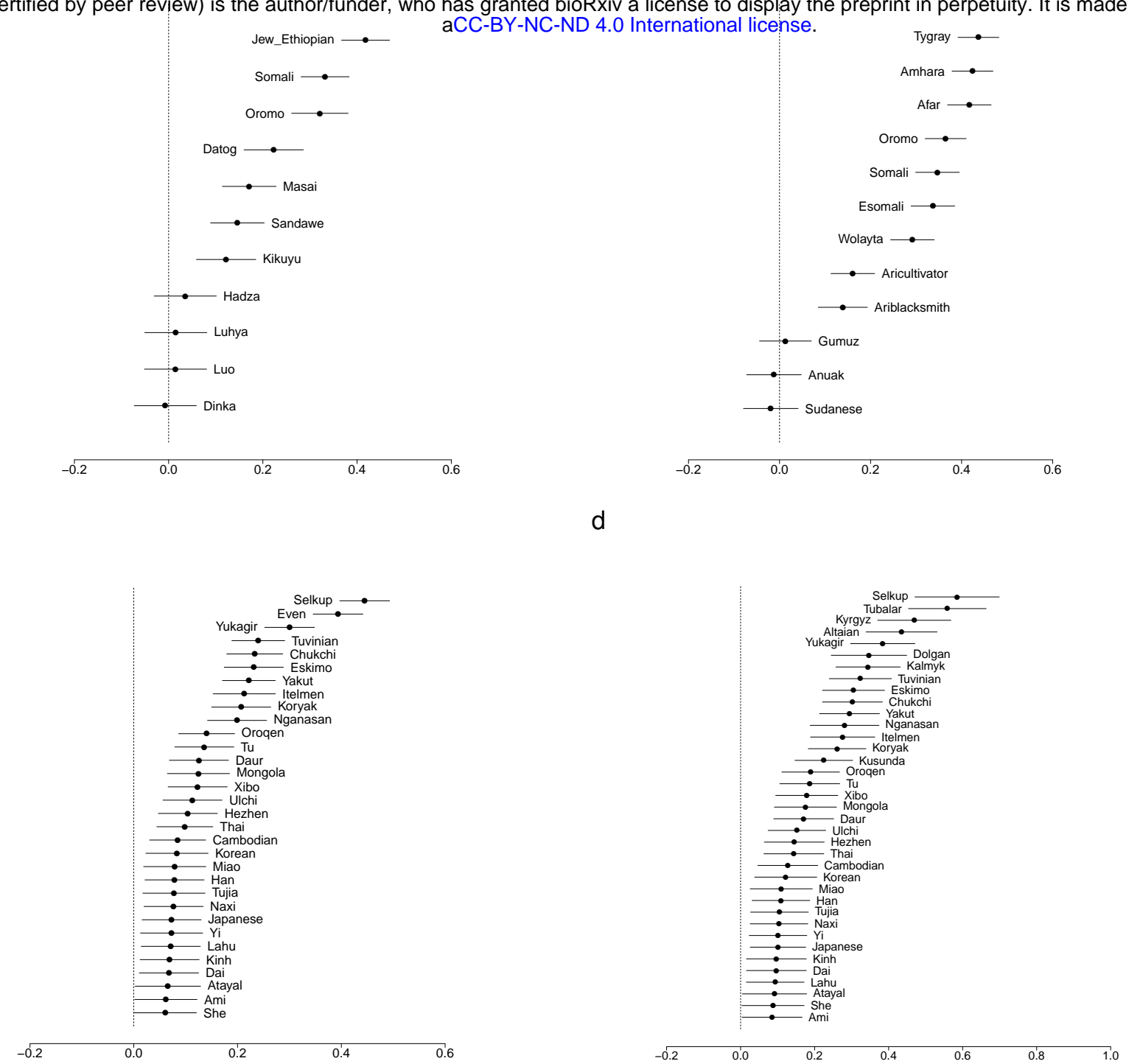

e

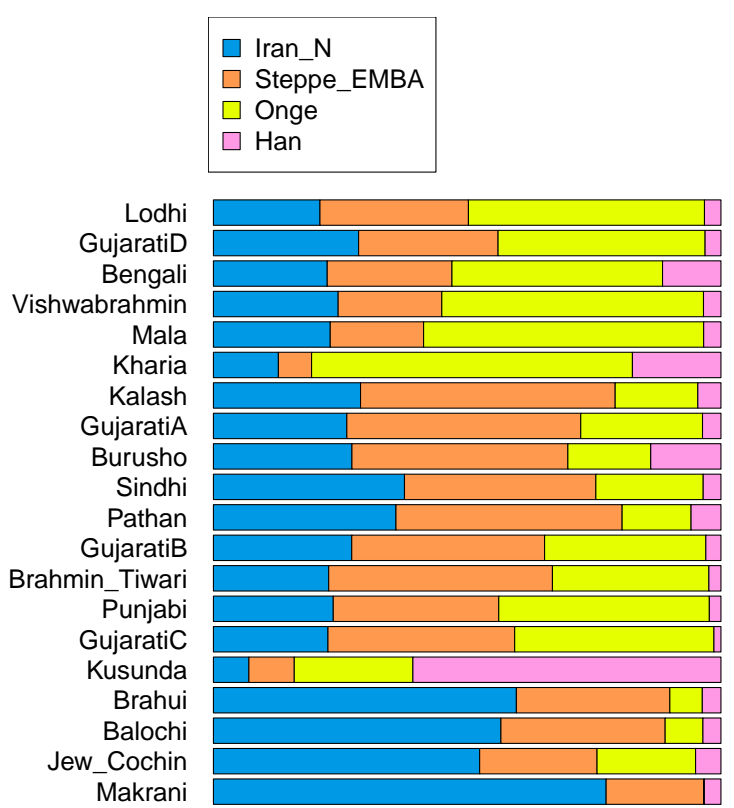




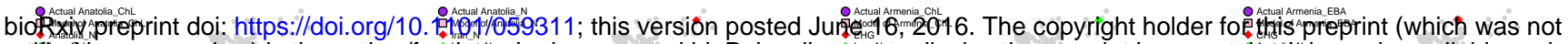
certified'by peer review) is the author/funder,", who has granted bioRxiv a licensel'to display the preprint in perpetuityo:lit' is made available under

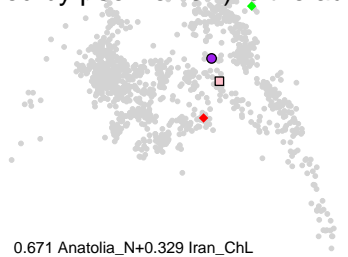

0.671 Anatolia_N+0.329 Iran_ChL

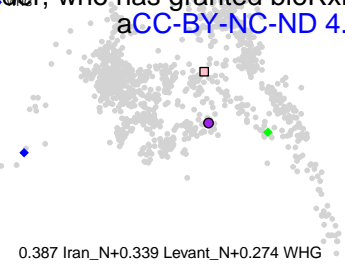

CHG

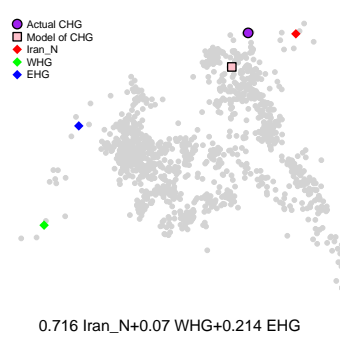

0.104 EHG+0.553 CHG+0.344 Anatolia_N

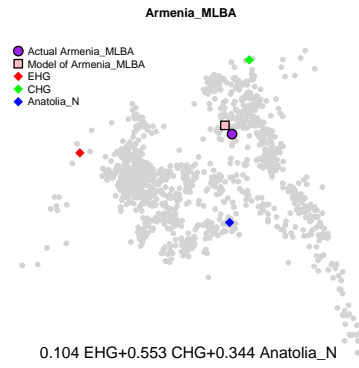

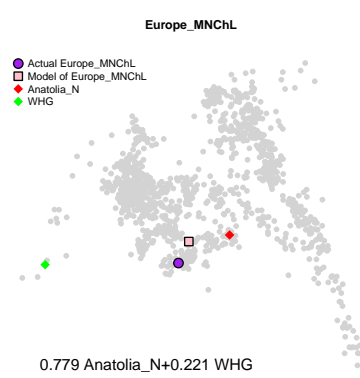
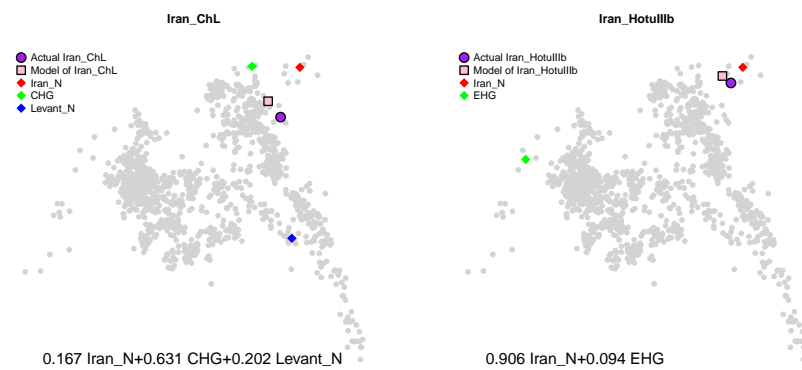

$0.906 \operatorname{Iran} \_\mathrm{N}+0.094 \mathrm{EHG}$

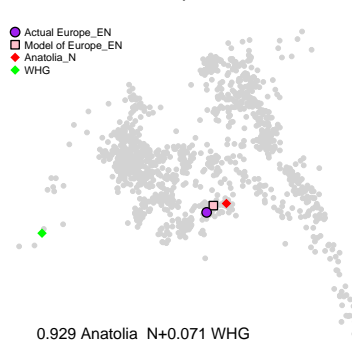

0.929 Anatolia $\_+0.071$ WHG

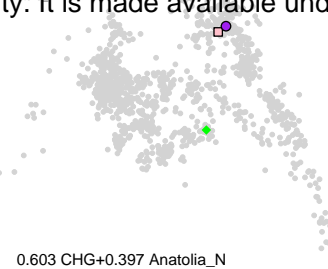

$0.603 \mathrm{CHG}+0.397$ Anatolia $\mathrm{N}$
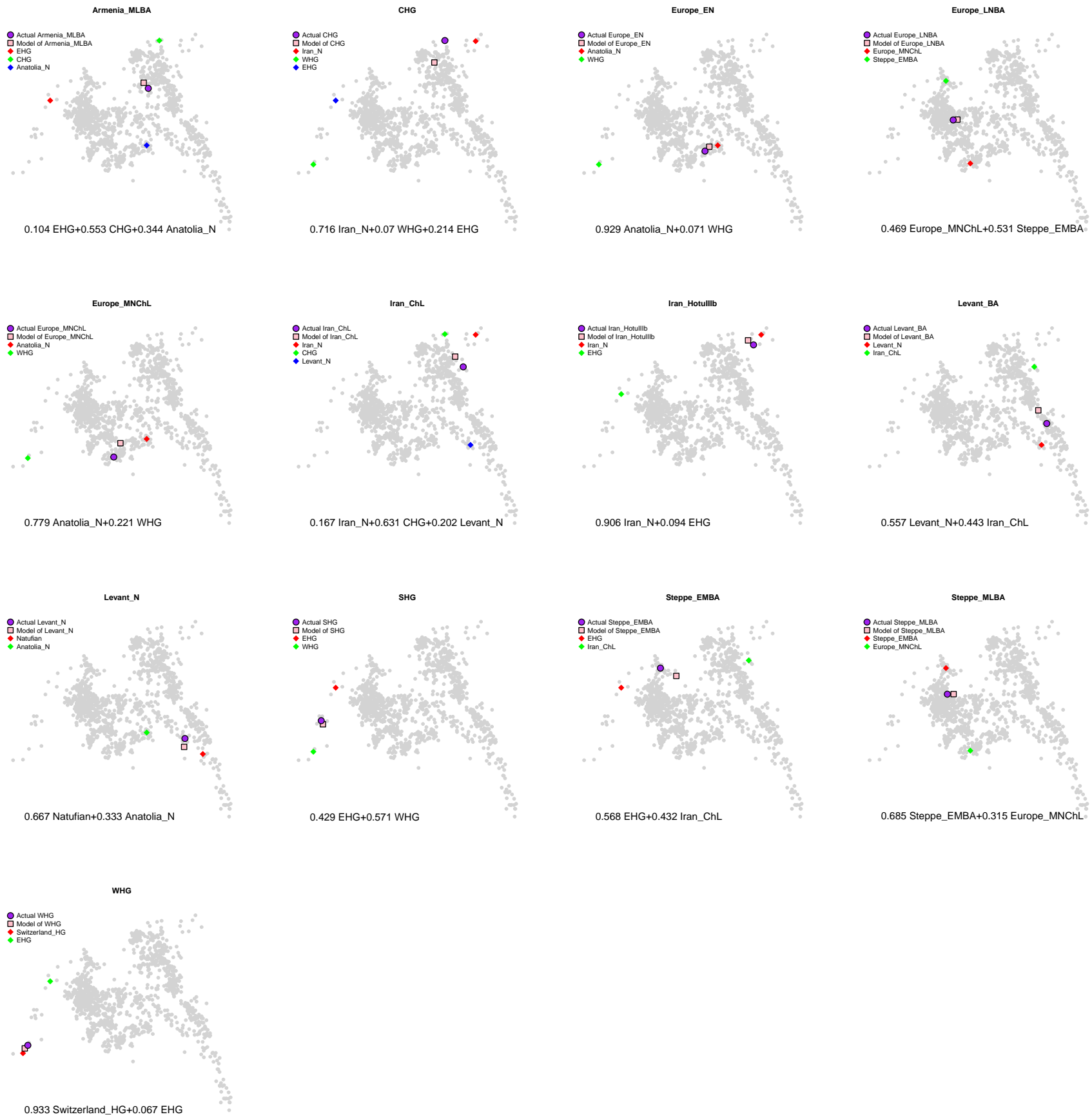

Extended Data Figure 5 

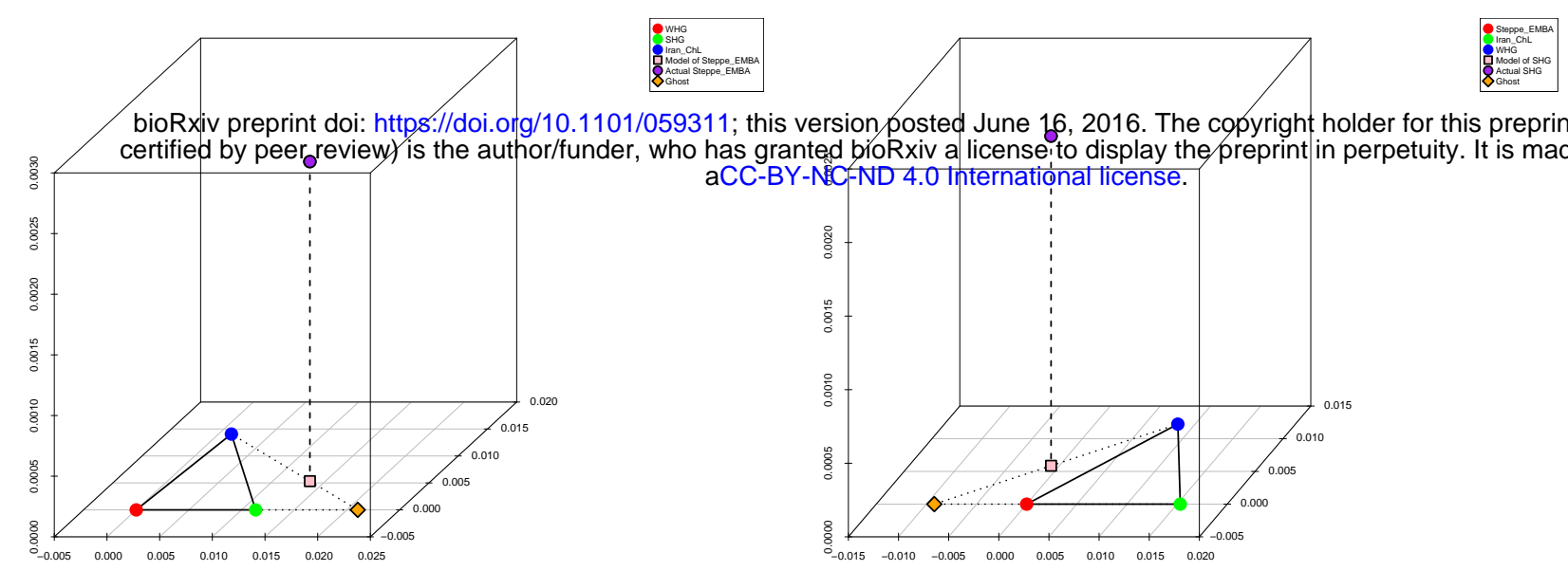

bioRxiv preprint doi: https://doi.org/10.1101/059311; this version posted June 16, 2016. The copyright holder for this preprint (which was not certified by peer reviewy) is the author/funder, who has granted bioRxiv a license to display the preprint in perpetuity. It is made available under

c

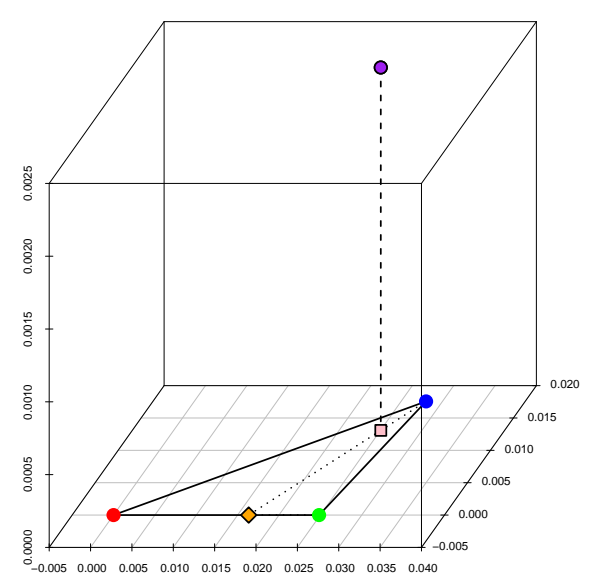

e

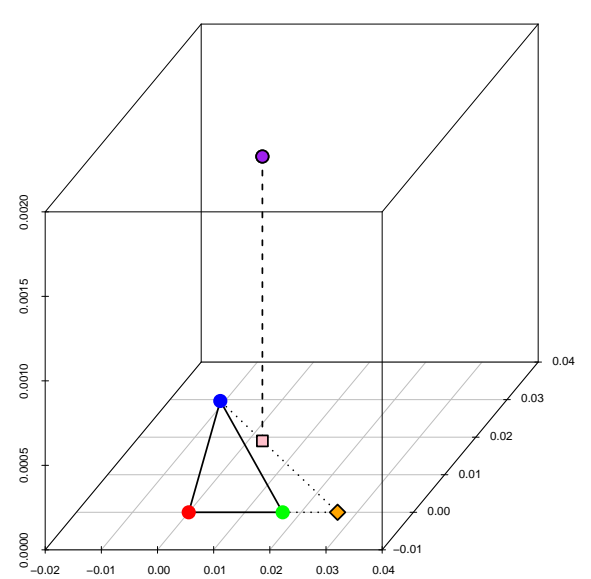
aCC-BY-NC-ND 4.0 Internatiónal license.

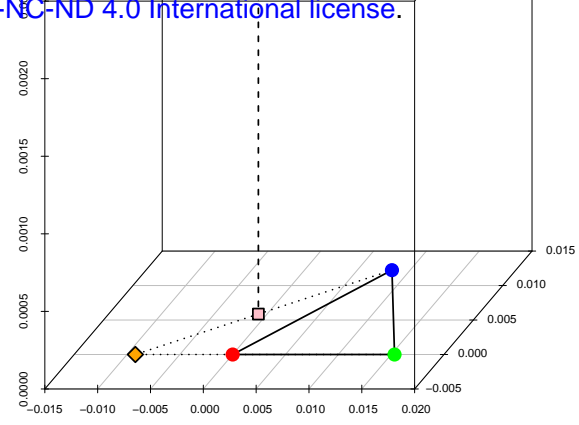

d
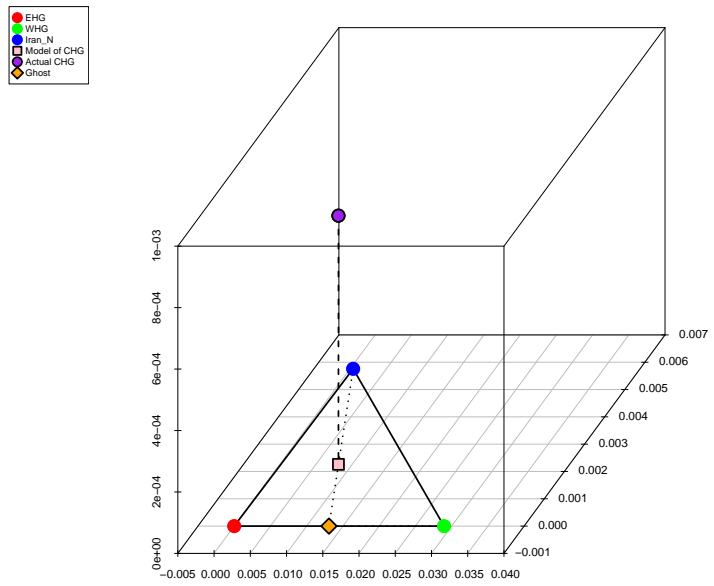

f

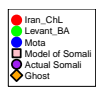

\section{Extended Data Figure 6}


Extended Data Figure 7

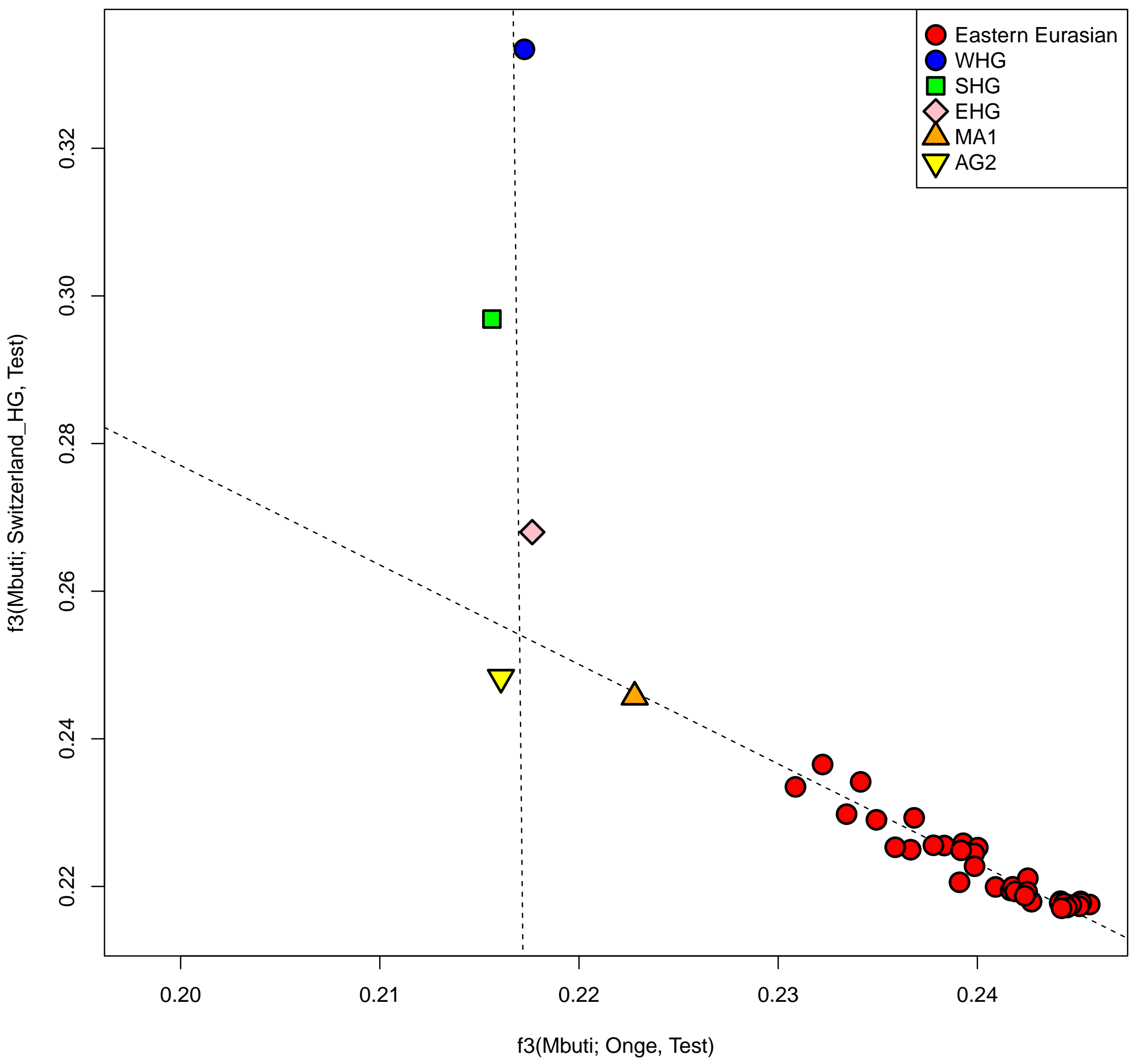

